Inventario dei manoscritti dell'Archivio Aldo Palazzeschi

a cura di

Simone Magherini 



\section{Carte d'autore}

01

Collana coordinata

dal Consiglio Direttivo del Centro di Studi «Aldo Palazzeschi» 
La collana «Carte d'autore» intende valorizzare e diffondere, in campo nazionale e internazionale, la pubblicazione in formato digitale (Open Access) di inventari, cataloghi, mostre, studi e ricerche promosse dal Centro di Studi "Aldo Palazzeschi" nell'ambito del progetto Carte d'autore online. 


\title{
Inventario dei manoscritti dell'Archivio Aldo Palazzeschi
}

\author{
a cura di \\ Simone Magherini
}

Società SfF Editrice Fiorentina 
(C) Copyright 2021 Centro di Studi «Aldo Palazzeschi» Licenza CC BY-NC-ND 4.0

E-Isbn: 978-88-6032-629-4

DOI: 10.35948/CSP/978-88-6032-629-4

Coordinamento archivistico ed editoriale: Manuela Ferraro Revisione dei dati delle schede digitali: Oleksandra Rekut-Liberatore Progetto e definizione dei flussi: Giovanni Salucci

Tracciato XML e standard archivistici: Manuela Ferraro, Giovanni Salucci Integrazione XML e Indesign: Arianna Marini

Progetto grafico e impaginazione: Francesco Sensoli

Progetto di copertina: Lorenzo Norfini

Piattaforma di gestione e procedure: Theke - Progettinrete 


\title{
Indice
}

\author{
VII Nota al testo \\ di Simone Magherini \\ XI Tavola delle sigle e delle abbreviazioni \\ Inventario dei manoscritti \\ dell'Archivio Aldo Palazzeschi \\ 3 Fondo Aldo Palazzeschi \\ 95 Fondo Acquisizioni Centro di Studi Aldo Palazzeschi \\ 99 Fondo "Carte del Signorino" \\ 103 Fondo Valentino Brosio \\ 105 Fondo Alberto Perrini
}





\section{Nota al testo}

Il presente lavoro intende fornire l'inventario dei manoscritti (serie Carte d'autore) conservati in cinque fondi dell'archivio del Centro di Studi «Aldo Palazzeschi» dell’Università di Firenze:

- Fondo "Aldo Palazzeschi»: donato per volontà testamentaria ${ }^{1}$ dallo scrittore alla Facoltà di Lettere dell'Università di Firenze (eredità acquisita il 13 settembre 1974);

- Fondo «Acquisizioni Centro di Studi Aldo Palazzeschi»: comprende le recenti carte acquistate dal Centro di Studi sul mercato antiquario a partire dal 2004;

- Fondo "Carte del Signorino": donato da Palazzeschi alla fedele domestica Plebe Bellocchio, acquistato sul mercato antiquario dalla Regione Toscana e concesso in uso al Centro di Studi nel luglio 2001;

- Fondo «Valentino Brosio»: donato dagli eredi Silvio e Marisa Montironi al Centro di Studi in data 7 settembre 2000;

- Fondo «Alberto Perrini»: donato da Alberto Perrini al Centro di Studi tra il 2002 e il 2003.

La parte più cospicua della documentazione è costituita dagli autografi palazzeschiani, a cui si aggiungono, nei fondi «Valentino Brosio» e «Alberto Perrini», alcuni scritti di Brosio, Perrini e Antoinette Riva (rielaborazioni di opere di Palazzeschi, autorizzate dallo scrittore o realizzate con la sua collaborazione).

Le serie Carte d'autore comprendono 220 fascicoli articolati in 464 unità documentarie, così ripartiti:

- 198 fascicoli e 438 unità documentarie nel Fondo «Aldo Palazzeschi»;

- 6 fascicoli e 7 unità documentarie nel Fondo «Acquisizioni Centro di Studi Aldo Palazzeschi»;

1 Il testamento olografo di Aldo Giurlani è stato sottoscritto in data 2 febbraio 1974, pubblicato in data 27 agosto 1974, n. 267658 di repertorio e registrato il 29 agosto 1974 al n. 9936, vol. 189, a rogito dott. Romualdo Manoni. 
- 9 fascicoli e 10 unità documentarie nel Fondo "Carte del Signorino";

- 2 fascicoli e 4 unità documentarie nel Fondo "Valentino Brosio»;

- 5 fascicoli e 5 unità documentarie nel Fondo «Alberto Perrini».

La documentazione copre un arco cronologico che va dall'esordio poetico di Palazzeschi, nel 1905, anno di pubblicazione della raccolta I cavalli bianchi, al 1974, anno della morte.

La scelta della data finale deriva dall'impossibilità di determinare un'ipotesi di datazione di alcuni autografi, non pubblicati: pertanto si è deciso di attribuire come termine ante quem la data della scomparsa dello scrittore.

Il materiale è organizzato per fascicoli, ciascuno contenente una o più unità documentarie. Fanno eccezione tre opere, Il palio dei buffi, Tutte le novelle, Nove sinfonie, articolate su 3 livelli: sottoserie (raccolta), fascicoli (novelle) e unità documentarie. La successione dei fascicoli segue l'ordinamento proposto nel corso dell'inventariazione dal gruppo di lavoro diretto da Ennio Bruschi $^{2}$.

Ogni serie dei manoscritti, identificata dal codice del livello, fornisce informazioni sul contenuto e sulla tipologia della documentazione, secondo l'esempio qui riportato:

\section{Serie AP 1.4}

Titolo: Carte d'autore

Consistenza: S19

Data: 1905 - 17 agosto 1974

Contiene i manoscritti palazzeschiani conservati dallo stesso scrittore.

L'attuale ordinamento, frutto di una prima riorganizzazione dei materiali effettuata nel corso del 2005 e completata nel 2021, rispetta il vincolo della Serie Carte d'autore quale è stata trasmessa ai curatori dell'archivio.

\section{Criteri di schedatura}

Di seguito si illustra un esempio di scheda utilizzata nell'inventario, con l'indicazione dei principali criteri di descrizione adottati:

FP3, 3.n

Titolo: «[invi]tando a seguirlo con la massima disin=voltura»

Tipologia documentaria: Manoscritto

Stato conservazione: anepigrafo, acefalo e mutilo

Consistenza: cc. 268, 270-288 + cc. 282 bis e 282tris, rif. di c. 282 (num. mod. 8-29)

Autore: Aldo Palazzeschi

Data: autunno 1967 - ottobre 1969

2 Cfr Manoscritti di Aldo Palazzeschi. Catalogo, a cura di Enio Bruschi, Premessa di Gino Tellini, Roma, Edizioni di Storia e Letteratura, Università degli Studi di Firenze, 2009. 
Nella descrizione della singola unità documentaria sono specificati il codice, il titolo, la tipologia documentaria, lo stato di conservazione (nel caso il manoscritto risultasse danneggiato), la consistenza, la data e l'autore.

La trascrizione dei titoli si è attenuta scrupolosamente all'originale, osservando i seguenti criteri:

[ ] le parentesi quadre indicano integrazioni basate sulla versione a stampa corrispondente

<> le parentesi uncinate riportano la porzione di testo barrato dall'autore

"» le virgolette basse sono utilizzate per la prima riga delle carte acefale

“" le virgolette alte segnalano un titolo virgolettato dall'autore

$=\quad$ il segno uguale è stato usato per completare la parola spezzata alla fine della prima riga

Sono stati compiuti interventi formali sul campo codice (che seguiva una sintassi irregolare), senza tuttavia stravolgere il nucleo del dato informativo.

AP 1.4.2 FP33, 33.b

$\mathrm{AP}$ 1.4.2. $\mathrm{FP} 3$, 3.e

$\mathrm{AP}$ 1.4.3. $\mathrm{FP} 32$

AP 1.4.3 FP32, 32

AP 1.4.1 FP37.22, 37.22.b

AP 1.4.1. FP 37.23

$$
\begin{array}{ll}
\rightarrow & \text { AP 1.4 FP33, 33.b } \\
\rightarrow & \text { AP 1.4 FP3, 3.e } \\
\rightarrow & \text { AP 1.4 FP32 } \\
\rightarrow & \text { AP 1.4 FP32, 32 } \\
\rightarrow & \text { AP 1.4 FP37.22, 37.22.b } \\
\rightarrow & \text { AP 1.4 FP37.23 }
\end{array}
$$

Per il campo data si è deciso di uniformare le oscillazioni dei valori registrati determinando gli estremi cronologici della prima e dell'ultima pubblicazione edita in vita dell'autore, non potendo stabilire con certezza, se i testi siano stati riveduti, revisionati o semplicemente ristampati.

Fanno eccezione, ove presenti, le dichiarazioni esplicite dello scrittore sull'avvio e sulle fasi di elaborazione di alcune opere ricavate dalle carte (in particolare dalle lettere) custodite nell'archivio Palazzeschi. Per i lavori inediti o pubblicati postumi, si fa riferimento a un arco temporale ampio che in alcuni casi si conclude con la morte dello scrittore. Per la risoluzione di altri problemi relativi alla datazione dei romanzi e delle poesie si è fatto ricorso agli apparati di Notizie sui testi a cura di Gino Tellinis ${ }^{3}$ e di Adele Dei ${ }^{4}$.

Simone MAGHeRINI

3 Gino Tellini, Notizie sui testi, in Aldo Palazzeschi, Tutti i romanzi, i e iI, a cura e con introduzione di Gino Tellini, Milano, Mondadori, 2005, I, pp. 1379-1671, e II, pp. 1367-1696.

4 Adele Dei, Notizie e note sui testi, in Aldo Palazzeschi, Tutte le poesie, a cura e con un saggio introduttivo di Adele Dei, Milano, Mondadori, 2002, pp. 941-1233. 



\section{Tavola delle sigle e delle abbreviazioni}

$\mathrm{AP}=$ Archivio «Aldo Palazzeschi»

$\mathrm{FP}=$ Fondo «Aldo Palazzeschi»

FBE $=$ Fondo "Carte del Signorino"

FBR $=$ Fondo «Valentino Brosio»

FPE $=$ Fondo «Alberto Perrini»

FPna $=$ Fondo «Acquisizioni Centro di Studi Aldo Palazzeschi»

ad int. di = specificazione utilizzata quando una o più carte non rappresen-

tano un rifacimento di altre precedenti, con uguale numerazione, ma una integrazione

c., cc. = carta, carte. (le carte si intendono di norma numerate dall'autore)

bis, tris = carte numerate dall'autore con numerazione bis e tris

err. rip. = carta la cui numerazione è erroneamente ripetuta. (indica un salto nella numerazione delle carte)

ex n. = precede vecchie segnature, in primis quella attribuita dal Catalogo Bettarini-Parenti ${ }^{1}$ ai manoscritti del Fondo «Aldo Palazzeschi»

$\mathrm{F}=$ fascicolo

manoscritto acefalo $=$ mancante, per guasto meccanico, della parte iniziale manoscritto anepigrafo $=$ mancante del titolo

manoscritto interrotto $=$ manoscritto non mutilo per guasto meccanico, ma la cui copiatura è stata interrotta dall'autore

manoscritto mutilo $=$ mancante, per guasto meccanico, di parti intermedie o della parte finale

num. err. $=$ carta erroneamente numerata

num. mod. $=$ carta numerata modernamente

p., pp. = pagina, pagine

$\mathrm{r} .=$ recto

rif. di / rif. del = specificazione utilizzata quando una o più carte numerate senza indicazione di bis o ter, rappresentano un rifacimento di altre precedenti

1 Rosanna Bettarini e Giovanni Parenti predisposero la prima catalogazione dei manoscritti, «poco dopo l'arrivo del prezioso corpus delle carte palazzeschiane», cfr. Manoscritti di Aldo Palazzeschi. Catalogo, cit., p. xIx. 
xı | Tavola delle sigle e delle abbreviazioni

$\mathrm{S}=$ scatola

s. n. = senza numerazione

$\mathrm{UD}=$ unità documentaria

v. $=$ verso

Nel licenziare il presente lavoro desidero esprimere un ringraziamento a coloro che in questi anni hanno contribuito alla schedatura, integrazione e revisione dei dati archivistici dei manoscritti dell'Archivio "Aldo Palazzeschi»: Barbara Silvia Anglani, Erika Bertelli, Stefania Alessandra Bottini, Enio Bruschi, Laura Diafani, Manuela Ferraro, Angela Frati, Irene Gambacorti, Sara Gelli, Francesca Mecatti, Oleksandra Rekut-Liberatore. 
Inventario dei manoscritti

dell'Archivio Aldo Palazzeschi 



\section{Fondo Aldo Palazzeschi}

\section{Serie AP 1.4}

Titolo: Carte d'autore

Consistenza: S19

Data: 1905 - 17 agosto 1974

Contiene i manoscritti palazzeschiani conservati dallo stesso scrittore. L'attuale ordinamento, frutto di una prima riorganizzazione dei materiali effettuata nel corso del 2005 e completata nel 2021, rispetta il vincolo della Serie Carte d'autore quale è stata trasmessa ai curatori dell'archivio.

FP1

Titolo: Un uomo a cavallo

Consistenza: UD3

Data: ante 1958 - 1964

FP1, 1.a

Titolo: Un uomo a cavallo

Tipologia documentaria: Manoscritto

Consistenza: cc. 1-8 + cc. 7-8, rif. di cc. 7-8 (num. mod. 7bis-8bis)

Autore: Palazzeschi, Aldo

Data: ante 1958 - 1964

FP1, 1.b

Titolo: Un uomo a cavallo

Tipologia documentaria: Manoscritto

Consistenza: cc. 1-8

Autore: Palazzeschi, Aldo

Data: ante 1958 - 1964

FP1, 1.c

Titolo: Il cavaliere bianco

Tipologia documentaria: Manoscritto

Consistenza: cc. 1-13

Autore: Palazzeschi, Aldo

Data: ante 1963 - 9 ottobre 1963

\section{FP2}

Titolo: Premessa a Cuor mio

Consistenza: UD1

Autore: Palazzeschi, Aldo

Data: ante 1968 - 1968 
FP2, 2

Titolo: "<Consentitemi, $>$ Prima d'incominciare,"

Tipologia documentaria: Manoscritto

Stato conservazione: anepigrafo

Consistenza: cc. 1-26

Autore: Palazzeschi, Aldo

Data: ante 1968 - 1968

FP3

Titolo: Stefanino

Consistenza: UD13

Data: autunno 1967 - ottobre 1969

FP3, 3.a

Titolo: «Da alcune mattine davanti alla»

Tipologia documentaria: Manoscritto

Stato conservazione: anepigrafo

Consistenza: cc. 1-144 + cc. 1-3, rif. di cc. 2-5 (num. mod. 1bis-3bis)

Autore: Palazzeschi, Aldo

Data: autunno 1967 - ottobre 1969

FP3, 3.b

Titolo: "Da alcune mattine davanti»

Tipologia documentaria: Manoscritto

Stato conservazione: anepigrafo

Consistenza: cc. 1-159 + c. 4, rif. di c. 5 (num. mod. 81)

Autore: Palazzeschi, Aldo

Data: autunno 1967 - ottobre 1969

FP3, 3.c

Titolo: «Nonostante che l'invito fosse per le»

Tipologia documentaria: Manoscritto

Consistenza: cc. 81-159, 162-172

Autore: Palazzeschi, Aldo

Data: autunno 1967 - ottobre 1969

FP3, 3.d

Titolo: «Dopo tante inutili attese, dopo»

Tipologia documentaria: Manoscritto

Consistenza: cc. 1-17

Autore: Palazzeschi, Aldo

Data: autunno 1967 - ottobre 1969

FP3, 3.e

Titolo: "Dopo tante inutili attese, dopo tante»

Tipologia documentaria: Manoscritto

Consistenza: cc. 1-18

Autore: Palazzeschi, Aldo

Data: autunno 1967 - ottobre 1969 
FP3, 3.f

Titolo: «Invece di calmare gli animi poneva»

Tipologia documentaria: Manoscritto

Stato conservazione: interrotto

Consistenza: cc. 1-8

Autore: Palazzeschi, Aldo

Data: autunno 1967 - ottobre 1969

FP3, 3.g

Titolo: Stefanino

Tipologia documentaria: Manoscritto

Consistenza: cc. 1-208 + c. 87bis (num. mod., ad int. di c. 88)

Autore: Palazzeschi, Aldo

Data: autunno 1967 - ottobre 1969

FP3, 3.h

Titolo: «Con sorprendente energia Stefa=nino»

Tipologia documentaria: Manoscritto

Consistenza: cc. 1-24

Autore: Palazzeschi, Aldo

Data: autunno 1967 - ottobre 1969

FP3, 3.i

Titolo: «Da alcune mattine davanti alla»

Tipologia documentaria: Manoscritto

Stato conservazione: anepigrafo e mutilo della c. 1

Consistenza: cc. 2-241

Autore: Palazzeschi, Aldo

Data: autunno 1967 - ottobre 1969

FP3, 3.1

Titolo: Stefanino

Tipologia documentaria: Manoscritto

Consistenza: cc. 1-257 (cc. 251-257 num. mod. 1-7)

Autore: Palazzeschi, Aldo

Data: autunno 1967 - ottobre 1969

FP3, 3.m

Titolo: «Ancora una volta bilanciando"

Tipologia documentaria: Manoscritto

Consistenza: c. 200

Autore: Palazzeschi, Aldo

Data: autunno 1967 - ottobre 1969

FP3, 3.n

Titolo: «[invi]tando a seguirlo con la massima disin=voltura» Tipologia documentaria: Manoscritto

Stato conservazione: anepigrafo, acefalo e mutilo

Consistenza: cc. 268, 270-288 + cc. 282bis e 282tris, rif. di c. 282 (num. mod. 8-29)

Autore: Palazzeschi, Aldo 
Data: autunno 1967 - ottobre 1969

FP3, 3.0

Titolo: «ed ai mezzi più adeguati per combat=terle»

Tipologia documentaria: Manoscritto

Stato conservazione: anepigrafo e acefalo

Consistenza: cc. 294-298 + cc. 294bis e 295bis, rif. di c. 294 e c. 295 (num. mod. 1-7)

Autore: Palazzeschi, Aldo

Data: autunno 1967 - ottobre 1969

FP4

Titolo: Marinetti e il futurismo

Consistenza: UD1

Data: ante 1968 - 1968

FP4, 4

Titolo: «In un pomeriggio di Gennaio»

Tipologia documentaria: Manoscritto

Stato conservazione: anepigrafo e mutilo

Consistenza: cc. 1-23, 25 e 27

Autore: Palazzeschi, Aldo

Data: ante 1968 - 1968

\section{FP5}

Titolo: La Piramide. Scherzo di cattivo genere e fuor di luogo

Consistenza: UD5

Data: anni Venti - 1926

FP5, 5.a

Titolo: A tre

Tipologia documentaria: Manoscritto

Stato conservazione: mutilo

Consistenza: cc. 1-6

Autore: Palazzeschi, Aldo

Data: anni Venti - 1926

FP5, 5.b

Titolo: A tre

Tipologia documentaria: Manoscritto

Consistenza: cc. 1-40 + c. 27, rif. di c. 27 (num. mod. 27bis)

Autore: Palazzeschi, Aldo

Data: anni Venti - 1926

FP5, 5.c

Titolo: <Duetto $>$ A due

Tipologia documentaria: Manoscritto

Consistenza: cc. 1-50 
Autore: Palazzeschi, Aldo

Data: anni Venti - 1926

FP5, 5.d

Titolo: A solo

Tipologia documentaria: Manoscritto

Consistenza: cc. 1-44 + 1 c. (num. mod. 4bis, ad int. di c. 4)

Autore: Palazzeschi, Aldo

Data: anni Venti - 1926

FP5, 5.e

Titolo: Venezia

Tipologia documentaria: Manoscritto

Consistenza: cc. 1-8 + c. 8 , rif. di c. 8 (num. mod. 8bis)

Autore: Palazzeschi, Aldo

Data: anni Venti - 1926

\section{FP6}

Titolo: Il pollo al diavolo

Consistenza: UD7

Data: post $1939-1967$

FP6, 6.a

Titolo: Il pollo alla diavola

Tipologia documentaria: Manoscritto

Consistenza: cc. 1-6

Autore: Palazzeschi, Aldo

Data: post 1939 - 15 ottobre 1939

FP6, 6.b

Titolo: Il pollo alla diavola

Tipologia documentaria: Manoscritto

Consistenza: cc. 1-6 + cc. 3 e 5, rif. di cc. 3 e 5 (num. mod. 3bis e 5 bis)

Autore: Palazzeschi, Aldo

Data: post 1939 - 15 ottobre 1939

FP6, 6.c

Titolo: Il pollo alla diavola

Tipologia documentaria: Manoscritto

Consistenza: cc. 1-6

Autore: Palazzeschi, Aldo

Data: post 1939 - 15 ottobre 1939

FP6, 6.d

Titolo: Il pollo al diavolo

Tipologia documentaria: Manoscritto

Consistenza: cc. 1-11

Autore: Palazzeschi, Aldo

Data: post $1939-1967$ 
FP6, 6.e

Titolo: il pollo al diavolo

Tipologia documentaria: Manoscritto

Consistenza: cc. 1-13

Autore: Palazzeschi, Aldo

Data: post $1939-1967$

FP6, 6.f

Titolo: il pollo al diavolo

Tipologia documentaria: Manoscritto

Consistenza: cc. 50-60 + c. 59, rif. di c. 59 (num. mod. 11)(num. mod. 1-12)

Autore: Palazzeschi, Aldo

Data: post 1939 - 1967

FP6, 6.g

Titolo: il pollo al diavolo

Tipologia documentaria: Manoscritto

Consistenza: cc. 50-61 (num. mod. 1-12)

Autore: Palazzeschi, Aldo

Data: post 1939 - 1967

FP7

Titolo: Usanze d'oggi

Consistenza: UD1

Data: ante 1960 - 27 marzo 1960

\section{FP7, 7}

Titolo: Usanze d'oggi

Tipologia documentaria: Manoscritto

Consistenza: cc. 1-8

Autore: Palazzeschi, Aldo

Data: ante 1960 - 27 marzo 1960

\section{FP8}

Titolo: Lo spogliarello

Consistenza: UD1

Data: ante 1960 - 1967

FP8, 8

Titolo: Lo spogliarello

Tipologia documentaria: Manoscritto

Consistenza: cc. 1-6

Autore: Palazzeschi, Aldo

Data: ante 1960 - 1967

\section{FP9}

Titolo: Serenate 
Consistenza: UD1

Data: ante 1960 - 1967

FP9, 9

Titolo: Serenate

Tipologia documentaria: Manoscritto Consistenza: cc. 41-43 (num. mod. 1-3) Autore: Palazzeschi, Aldo

Data: ante 1960 - 1967

\section{FP10}

Titolo: Usanze d'oggi

Consistenza: UD1

Data: ante $1959-1^{\circ}$ novembre 1959

FP10, 10

Titolo: Usanze d'oggi

Tipologia documentaria: Manoscritto

Consistenza: cc. 1-8

Autore: Palazzeschi, Aldo

Data: ante $1959-1^{\circ}$ novembre 1959

\section{FP11}

Titolo: I paggetti del 900

Consistenza: UD1

Data: ante 1959 - 1967

FP11, 11

Titolo: I paggetti del 900

Tipologia documentaria: Manoscritto Consistenza: cc. 1-3

Autore: Palazzeschi, Aldo

Data: ante 1959 - 1967

\section{FP12}

Titolo: Dolore

Consistenza: UD1

Data: post $1959-1967$

FP12, 12

Titolo: dolore

Tipologia documentaria: Manoscritto

Consistenza: cc. 1-4

Autore: Palazzeschi, Aldo

Data: post 1959 - 1967 
FP13

Titolo: Marco

Consistenza: UD1

Data: ante 1959 - 1967

FP13, 13

Titolo: Marco

Tipologia documentaria: Manoscritto

Consistenza: cc. 1-5

Autore: Palazzeschi, Aldo

Data: ante 1959 - 1967

\section{FP14}

Titolo: La borsetta

Consistenza: UD1

Data: ante 1959 - 1967

FP14, 14

Titolo: La borsetta

Tipologia documentaria: Manoscritto Consistenza: cc. 1-4 (num. mod.)

Autore: Palazzeschi, Aldo

Data: ante 1959 - 1967

\section{FP15}

Titolo: Il domicilio della bellezza

Consistenza: UD4

Data: ante 1940 - 1967

\section{FP15, 15.a}

Titolo: bellezza

Tipologia documentaria: Manoscritto Consistenza: cc. 1-7 (num. mod. 1-8)

Autore: Palazzeschi, Aldo

Data: ante 1940 - 1967

FP15, 15.b

Titolo: bellezza

Tipologia documentaria: Manoscritto Consistenza: cc. 1-7 (num. mod. 1-7)

Autore: Palazzeschi, Aldo

Data: ante 1940 - 1967

FP15, 15.C

Titolo: «Non pochi, specialmente fra i poeti» Tipologia documentaria: Manoscritto

Consistenza: cc. 1-7

Autore: Palazzeschi, Aldo 
Data: ante $1940-1967$

\section{FP15, 15.d}

Titolo: bellezza

Tipologia documentaria: Manoscritto

Consistenza: cc. 11-18 + 6 cc. (num. d'autore da «18 $2^{\circ} »$ a «18 $6^{\circ} »$ e num. mod. 1-13)

Autore: Palazzeschi, Aldo

Data: ante 1940 - 1967

\section{FP16}

Titolo: Le città del silenzio

Consistenza: UD5

Data: ante 1940 - 1967

FP16, 16.a

Titolo: le città del silenzio

Tipologia documentaria: Manoscritto

Consistenza: cc. 1-7

Autore: Palazzeschi, Aldo

Data: ante 1940 - 1967

FP16, 16.b

Titolo: le città del silenzio

Tipologia documentaria: Manoscritto

Consistenza: cc. 29-30 (num. mod. 1-2)

Autore: Palazzeschi, Aldo

Data: ante 1940 - 1967

FP16, 16.c

Titolo: le città del silenzio

Tipologia documentaria: Manoscritto

Consistenza: cc. 29-38 (num. mod. 1-10)

Autore: Palazzeschi, Aldo

Data: ante 1940 - 1967

\section{FP16, 16.d}

Titolo: le città del silenzio

Tipologia documentaria: Manoscritto

Consistenza: cc. 29-31 (num. mod. 1-3)

Autore: Palazzeschi, Aldo

Data: ante 1940 - 1967

FP16, 16.e

Titolo: le città del silenzio

Tipologia documentaria: Manoscritto

Consistenza: cc. 29-39 + 3 cc. (num. d'autore da «39bis» a «39 $4^{\circ}$ » e num. mod. 1-14)

Autore: Palazzeschi, Aldo

Data: ante 1940 - 1967 
FP17

Titolo: Moda

Consistenza: UD2

Data: ante 1961 - 2 luglio 1961

\section{FP17, 17.a}

Titolo: «come un vecchio ed eroico soldatone alto»

Tipologia documentaria: Manoscritto

Consistenza: 5 cc. (tutte num. d'autore come c. 8, rif. di c. 8)

Autore: Palazzeschi, Aldo

Data: ante 1961 - 2 luglio 1961

FP17, 17.b

Titolo: Moda

Tipologia documentaria: Manoscritto

Consistenza: cc. 1-7

Autore: Palazzeschi, Aldo

Data: ante 1961 - 2 luglio 1961

\section{FP18}

Titolo: Finanza

Consistenza: UD2

Data: ante 1961 - 1967

FP18, 18.a

Titolo: "protesi e sempre più sbracciando e sorridendo" Tipologia documentaria: Manoscritto

Stato conservazione: anepigrafo, acefalo e mutilo

Consistenza: 1 c. s. n.

Autore: Palazzeschi, Aldo

Data: ante 1961 - 1967

\section{FP18, 18.b}

Titolo: «Essendo io figliolo di mercante all'uso antico»

Tipologia documentaria: Manoscritto

Stato conservazione: anepigrafo e mutilo

Consistenza: cc. 1-2

Autore: Palazzeschi, Aldo

Data: ante 1961 - 1967

FP18, 18.c

Titolo: Finanza

Tipologia documentaria: Manoscritto

Consistenza: cc. 1-7

Autore: Palazzeschi, Aldo

Data: ante 1961 - 1967 


\section{FP19}

Titolo: Palazzeschi: quasi un Codice di Perelà

Consistenza: UD1

Data: ante 1965 - 11 luglio 1965

FP19, 19

Titolo: A proposito di premi

Tipologia documentaria: Manoscritto

Consistenza: cc. 1-8

Autore: Palazzeschi, Aldo

Data: ante 1965 - 11 luglio 1965

\section{FP20}

Titolo: Saint-Germain-des-Prés

Consistenza: UD1

Data: ante 1955 - 1957

FP20, 20

Titolo: Saint-Germain des Prés

Tipologia documentaria: Manoscritto

Stato conservazione: mutilo

Consistenza: cc. 1-4

Autore: Palazzeschi, Aldo

Data: ante 1955 - 1957

\section{FP21}

Titolo: Il Controdolore

Consistenza: UD1

Data: post 1914 - 1956

\section{FP21, 21}

Titolo: «Se credete che sia profondo quello che comunemente» Tipologia documentaria: Manoscritto Stato conservazione: anepigrafo, acefalo e mutilo Consistenza: cc. 51-64 (num. mod. 1-14) Autore: Palazzeschi, Aldo

Data: post 1914 - 1956

\section{FP22}

Titolo: L'ultima margherita

Consistenza: UD1

Data: ante 1945 - 1964

FP22, 22

Titolo: L'ultima margherita

Tipologia documentaria: Manoscritto

Stato conservazione: mutilo 
Consistenza: cc. 1-6

Autore: Palazzeschi, Aldo

Data: ante 1945 - 1964

FP23

Titolo: Grandi firme all'asta

Consistenza: UD1

Data: ante 1930 - 1964

FP23, 23

Titolo: Grandi firme

Tipologia documentaria: Manoscritto

Consistenza: cc. 1-7

Autore: Palazzeschi, Aldo

Data: ante 1930 - 1964

\section{FP24}

Titolo: Pettegolezzi in Paradiso

Consistenza: UD1

Data: ante 1956 - 1964

FP24, 24

Titolo: Pettegolezzi in Paradiso

Tipologia documentaria: Manoscritto

Stato conservazione: mutilo

Consistenza: cc. 1-8 (c. 8 num. mod.)

Autore: Palazzeschi, Aldo

Data: ante 1956 - 1964

\section{FP25}

Titolo: Scompartimento per signore sole Consistenza: UD5

Data: ante 1957-1964

\section{FP25, 25.a}

Titolo: Scompartimento per signore sole Tipologia documentaria: Manoscritto Consistenza: cc. 1-7

Autore: Palazzeschi, Aldo

Data: ante 1957 - 1964

FP25, 25.b

Titolo: Scompartimento per signore sole Tipologia documentaria: Manoscritto Consistenza: cc. 1-8

Autore: Palazzeschi, Aldo

Data: ante 1957 - 1964 
FP25, 25.c

Titolo: Scompartimento per signore sole

Tipologia documentaria: Manoscritto

Consistenza: cc. 1-8

Autore: Palazzeschi, Aldo

Data: ante $1957-1964$

FP25, 25.d

Titolo: Scompartimento per signore sole

Tipologia documentaria: Manoscritto

Consistenza: cc. 1-8

Autore: Palazzeschi, Aldo

Data: ante 1957 - 1964

FP25, 25.e

Titolo: Scompartimento per signore sole

Tipologia documentaria: Manoscritto

Consistenza: cc. 1-12 (num. d'autore a lapis 25-36)

Autore: Palazzeschi, Aldo

Data: ante 1957- 1964

\section{FP26}

Titolo: Il giuramento dei riformati

Consistenza: UD3

Data: ante 1920 - 1964

FP26, 26.a

Titolo: Il giuramento dei riformati

Tipologia documentaria: Manoscritto

Stato conservazione: mutilo

Consistenza: cc. 1-7

Autore: Palazzeschi, Aldo

Data: ante 1955 - 1964

FP26, 26.b

Titolo: Il giuramento dei riformati

Tipologia documentaria: Manoscritto

Consistenza: cc. $1-10+2$ cc. 10 , rif. di c. 10

Autore: Palazzeschi, Aldo

Data: ante 1955- 1964

FP26, 26.c

Titolo: «Da pochi giorni ero rientrato al reggimento e qualche»

Tipologia documentaria: Manoscritto

Stato conservazione: anepigrafo e mutilo

Consistenza: cc. 95-99 (num. mod. 4, 6, 8, 11 e 12)

Autore: Palazzeschi, Aldo

Data: ante 1920 - 1964 
FP27

Titolo: «Il giorno 16 Luglio dell'anno 1916 alle otto»

Consistenza: UD1

Data: ante 1920 - 1964

FP27, 27

Titolo: «Il giorno 16 Luglio dell'anno 1916 alle otto»

Tipologia documentaria: Manoscritto

Stato conservazione: anepigrafo

Consistenza: cc. 2-16 + c. 14, rif. di c. 14

Autore: Palazzeschi, Aldo

Data: ante 1920 - 1964

\section{FP28}

Titolo: "In Santa Maria delle Grazie un fanciullo mi»

Consistenza: UD1

Data: ante 1920 - 1964

FP28, 28

Titolo: «In Santa Maria delle Grazie un fanciullo mi»

Tipologia documentaria: Manoscritto

Stato conservazione: anepigrafo e mutilo

Consistenza: c. 91 e c. 93 + c. 93 , rif. di c. 93

Autore: Palazzeschi, Aldo

Data: ante 1920 - 1964

\section{FP29}

Titolo: "Gli operai d'ogni genere e colore finivano per trovarvi»

Consistenza: UD1

Data: ante 1920 - 1964

\section{FP29, 29}

Titolo: «Gli operai d'ogni genere e colore finivano per trovarvi»

Tipologia documentaria: Manoscritto

Stato conservazione: anepigrafo

Consistenza: cc. 52-55 (num. mod. 1-4)

Autore: Palazzeschi, Aldo

Data: ante 1920 - 1964

\section{FP30}

Titolo: "Allo stesso modo che a Firenze tutto volgeva al tra=gico" Consistenza: UD1

Data: ante 1920 - 1964

FP30, 30

Titolo: "Allo stesso modo che a Firenze tutto volgeva al tra=gico" Tipologia documentaria: Manoscritto 
Stato conservazione: anepigrafo

Consistenza: cc. 101-105 (num. mod. 15-19)

Autore: Palazzeschi, Aldo

Data: ante 1920 - 1964

\section{FP31}

Titolo: «Se il mio naturale carattere mi avesse seguito dentro»

Consistenza: UD1

Data: ante 1920 - 1964

\section{FP31, 31}

Titolo: «Se il mio naturale <spirito> carattere mi avesse seguito dentro»

Tipologia documentaria: Manoscritto

Stato conservazione: anepigrafo

Consistenza: cc. 17-25

Autore: Palazzeschi, Aldo

Data: ante 1920 - 1964

\section{FP32}

Titolo: «Da pochi giorni ero entrato al reggimento allor=quando»

Consistenza: UD1

Data: ante 1920 - 1964

FP32, 32

Titolo: «Da pochi giorni ero rientrato al reggimento allor=quando"

Tipologia documentaria: Manoscritto

Stato conservazione: anepigrafo

Consistenza: cc. 95-100 (num. mod. 5, 7, 9-10, 13-14)

Autore: Palazzeschi, Aldo

Data: ante 1920 - 1964

\section{FP33}

Titolo: Piazza della Libertà

Consistenza: UD7

Data: ante 1974 - marzo-giugno 1974

FP33, 33.a

Titolo: Alle ore 18 Comizio

Tipologia documentaria: Manoscritto

Stato conservazione: acefalo, anepigrafo e mutilo

Consistenza: cc. 1-7 + c. 5 , rif. di c. 5

Autore: Palazzeschi, Aldo

Data: ante 1974 - marzo-giugno 1974

FP33, 33.b

Titolo: Piazza della Libertà

Tipologia documentaria: Manoscritto 
Consistenza: cc. 1-26

Autore: Palazzeschi, Aldo

Data: ante 1974 - marzo-giugno 1974

FP33, 33.c

Titolo: Piazza della Libertà

Tipologia documentaria: Manoscritto

Stato conservazione: mutilo

Consistenza: cc. 1-43

Autore: Palazzeschi, Aldo

Data: ante 1974 - marzo-giugno 1974

FP33, 33.d

Titolo: Piazza della Libertà

Tipologia documentaria: Manoscritto

Consistenza: cc. 1-51

Autore: Palazzeschi, Aldo

Data: ante 1974 - marzo-giugno 1974

FP33, 33.e

Titolo: «Ma visto che il tanto atteso getto <dal>» Tipologia documentaria: Manoscritto Stato conservazione: acefalo, anepigrafo e mutilo Consistenza: cc. 19, 22, 44 e 44 (num. mod. 1-4) Autore: Palazzeschi, Aldo

Data: ante 1974 - marzo-giugno 1974

FP33, 33.f

Titolo: Piazza della Libertà

Tipologia documentaria: Manoscritto

Consistenza: cc. 1-83

Autore: Palazzeschi, Aldo

Data: ante 1974 - marzo-giugno 1974

FP33, 33.9

Titolo: Piazza della Libertà

Tipologia documentaria: Manoscritto

Consistenza: cc. 1-83

Autore: Palazzeschi, Aldo

Data: ante 1974 - marzo-giugno 1974

FP34

Titolo: La sora Corilla

Consistenza: UD2

Data: ante 1954 - 1964

FP34, 34.a

Titolo: La signora Corilla

Tipologia documentaria: Manoscritto 
Consistenza: cc. 1-13 (num. mod. 12-24)

Autore: Palazzeschi, Aldo

Data: ante 1954 - 1957

\section{FP34, 34.b}

Titolo: "di capire e discutere; posta difronte alla realtà si chiu=deva» Tipologia documentaria: Manoscritto

Stato conservazione: acefalo e anepigrafo

Consistenza: cc. 2-8 (num. mod. 1-7)

Autore: Palazzeschi, Aldo

Data: ante 1954 - 1964

\section{FP35}

Titolo: La mia no. Mentre dormo, la mia anima lavora

Consistenza: UD1

Data: 1905 - 17 agosto 1974

\section{FP35, 35}

Titolo: La mia no. Mentre <io> dormo, la mia anima lavora

Tipologia documentaria: Manoscritto

Stato conservazione: anepigrafo

Consistenza: cc. 1-3 (c. 3 s. n.)

Autore: Palazzeschi, Aldo

Data: 1905 - 17 agosto 1974 


\section{- FP36}

Titolo: Il palio dei buffi

Consistenza: F17

Data: ante 1912 - 1957

\section{FP36.1}

Titolo: Lo zio e il nipote

Consistenza: UD4

Data: ante 1933 - 1957

FP36.1, 36.1.a

Titolo: Zio e nipote

Tipologia documentaria: Manoscritto

Consistenza: cc. 1-46

Autore: Palazzeschi, Aldo

Data: ante 1933-1957

FP36.1, 36.1.b

Titolo: Zio e nipote

Tipologia documentaria: Manoscritto

Stato conservazione: mutilo

Consistenza: cc. 1-25

Autore: Palazzeschi, Aldo

Data: ante 1933 - 1957

FP36.1, 36.1.c

Titolo: "L'anima a Dio, il corpo alla terra, la roba a»

Tipologia documentaria: Manoscritto

Stato conservazione: acefalo e mutilo

Consistenza: cc. 18-38 (num. d'autore soprascritta a precedente da 25 a 45 e num. mod. 1-21)

Autore: Palazzeschi, Aldo

Data: ante 1933 - 1957

FP36.1, 36.1.d

Titolo: <Zio e nipote> Lo zio e il nipote

Tipologia documentaria: Manoscritto

Consistenza: cc. 1-49 + cc. 47-49 (num. d'autore soprascritta a precedente da 39 a 41 e num. mod. 22-24)

Autore: Palazzeschi, Aldo

Data: ante 1933 - 1957

\section{FP36.2}

Titolo: Carburo e Birchio

Consistenza: UD4

Data: ante 1932 - 1957 
FP36.2, 36.2.a

Titolo: Carburo e Birchio

Tipologia documentaria: Manoscritto

Consistenza: cc. 1-7

Autore: Palazzeschi, Aldo

Data: ante $1932-1957$

FP36.2, 36.2.b

Titolo: Carburo e Birchio

Tipologia documentaria: Manoscritto

Consistenza: cc. 1-8

Autore: Palazzeschi, Aldo

Data: ante $1932-1957$

FP36.2, 36.2.c

Titolo: Carburo e Birchio

Tipologia documentaria: Manoscritto

Consistenza: cc. 1-8

Autore: Palazzeschi, Aldo

Data: ante $1932-1957$

FP36.2, 36.2.d

Titolo: Carburo e Birchio

Tipologia documentaria: Manoscritto Consistenza: cc. 1-9

Autore: Palazzeschi, Aldo

Data: ante 1932 - 1957

\section{FP36.3}

Titolo: Il gobbo

Consistenza: UD1

Data: ante 1912 - 1957

FP36.3, 36.3

Titolo: Il gobbo

Tipologia documentaria: Manoscritto

Consistenza: cc. 1-15

Autore: Palazzeschi, Aldo

Data: ante 1912 - 1957

\section{FP36.4}

Titolo: La gloria

Consistenza: UD5

Data: ante 1926 - 1957

FP36.4, 36.4.a

Titolo: La Gloria

Tipologia documentaria: Manoscritto 
Stato conservazione: mutilo

Consistenza: cc. 1-9 + c. 7bis (num. mod.) ad intervallo di c. 9

Autore: Palazzeschi, Aldo

Data: ante 1926 - 1957

FP36.4, 36.4.b

Titolo: «Uno. Due. Tre. Quattro. Cinque. Sei.» Tipologia documentaria: Manoscritto

Stato conservazione: anepigrafo

Consistenza: cc. 1-9

Autore: Palazzeschi, Aldo

Data: ante 1926 - 1957

FP36.4, 36.4.c

Titolo: La gloria

Tipologia documentaria: Manoscritto

Consistenza: cc. 1-10

Autore: Palazzeschi, Aldo

Data: ante 1926 - 1957

FP36.4, 36.4.d

Titolo: La gloria

Tipologia documentaria: Manoscritto

Consistenza: cc. 1-9

Autore: Palazzeschi, Aldo

Data: ante 1926 - 1957

FP36.4, 36.4.e

Titolo: La gloria

Tipologia documentaria: Manoscritto

Consistenza: cc. 1-9

Autore: Palazzeschi, Aldo

Data: ante 1926 - 1957

\section{FP36.5}

Titolo: Pochini e Tamburini

Consistenza: UD1

Data: ante 1929 - 1957

FP36.5, 36.5

Titolo: Pochini e Tamburini

Tipologia documentaria: Manoscritto

Consistenza: cc. 1-5

Autore: Palazzeschi, Aldo

Data: ante 1929 - 1957

\section{FP36.6}

Titolo: Il dono 


\section{Consistenza: UD4}

Data: ante 1937 - 1957

FP36.6, 36.6.a

Titolo: Il dono

Tipologia documentaria: Manoscritto

Consistenza: cc. 1-18

Autore: Palazzeschi, Aldo

Data: ante 1937 - 1957

FP36.6, 36.6.b

Titolo: Il dono

Tipologia documentaria: Manoscritto

Consistenza: cc. 1-30 (c. 25 sul v. di c. 24)

Autore: Palazzeschi, Aldo

Data: ante 1937 - 1957

FP36.6, 36.6.c

Titolo: Il dono

Tipologia documentaria: Manoscritto

Consistenza: cc. 1-23 + cc. 21-24, rif. di cc. 20-24 (num. mod. c. 24 e 1-4)

Autore: Palazzeschi, Aldo

Data: ante 1937 - 1957

FP36.6, 36.6.d

Titolo: Il dono

Tipologia documentaria: Manoscritto

Consistenza: cc. 1-25

Autore: Palazzeschi, Aldo

Data: ante 1937 - 1957

\section{FP36.7}

Titolo: Gedeone e la sua Stella

Consistenza: UD3

Data: ante 1932 - 1957

FP36.7, 36.7.a

Titolo: Gedeone e la sua Stella

Tipologia documentaria: Manoscritto

Consistenza: cc. 1-7

Autore: Palazzeschi, Aldo

Data: ante $1932-1957$

FP36.7, 36.7.b

Titolo: Gedeone e la sua Stella

Tipologia documentaria: Manoscritto

Consistenza: cc. 1-8

Autore: Palazzeschi, Aldo

Data: ante 1932 - 1957 
FP36.7, 36.7.c

Titolo: Gedeone e la sua Stella

Tipologia documentaria: Manoscritto

Consistenza: cc. 1-8

Autore: Palazzeschi, Aldo

Data: ante 1932 - 1957

\section{FP36.8}

Titolo: Vita

Consistenza: UD3

Data: ante 1934 - 1957

\section{FP36.8, 36.8.a}

Titolo: Vita

Tipologia documentaria: Manoscritto

Stato conservazione: mutilo

Consistenza: cc. 1-17

Autore: Palazzeschi, Aldo

Data: ante 1934 - 1957

FP36.8, 36.8.b

Titolo: Vita

Tipologia documentaria: Manoscritto

Stato conservazione: mutilo

Consistenza: cc. 1-14

Autore: Palazzeschi, Aldo

Data: ante 1934 - 1957

FP36.8, 36.8.c

Titolo: Vita

Tipologia documentaria: Manoscritto

Stato conservazione: mutilo

Consistenza: cc. 1-21

Autore: Palazzeschi, Aldo

Data: ante 1934 - 1957

\section{FP36.9}

Titolo: Il punto nero

Consistenza: UD5

Data: ante $1937-1957$

\section{FP36.9, 36.9.a}

Titolo: «un'indiscrezione del genere potrebbe farlo ripetere. A» Tipologia documentaria: Manoscritto Stato conservazione: acefalo e anepigrafo

Consistenza: cc. 17-18 (num. mod. 1-2)

Autore: Palazzeschi, Aldo

Data: ante 1937 - 1957 
FP36.9, 36.9.b

Titolo: "essi stessi compivano quel lavoro del giorno" Tipologia documentaria: Manoscritto Stato conservazione: anepigrafo, acefalo e mutilo Consistenza: cc. 9-18 (num. mod. 1-10)

Autore: Palazzeschi, Aldo

Data: ante 1937 - 1957

\section{FP36.9, 36.9.c}

Titolo: Un punto nero

Tipologia documentaria: Manoscritto

Stato conservazione: interrotto

Consistenza: cc. 1-16

Autore: Palazzeschi, Aldo

Data: ante 1937 - 1957

FP36.9, 36.9.d

Titolo: Un punto nero

Tipologia documentaria: Manoscritto

Consistenza: cc. 1-16

Autore: Palazzeschi, Aldo

Data: ante 1937 - 1957

FP36.9, 36.9.e

Titolo: Il punto nero

Tipologia documentaria: Manoscritto Consistenza: cc. 1-19

Autore: Palazzeschi, Aldo

Data: ante 1937 - 1957

\section{FP36.10}

Titolo: Amore

Consistenza: UD6

Data: ante 1929 - 1957

FP36.10, 36.10.a

Titolo: Amore

Tipologia documentaria: Manoscritto

Consistenza: cc. 1-7

Autore: Palazzeschi, Aldo

Data: ante 1929 - 1957

FP36.10, 36.10.b

Titolo: Amore

Tipologia documentaria: Manoscritto

Consistenza: cc. 1-5

Autore: Palazzeschi, Aldo

Data: ante 1929- 1957 
FP36.10, 36.10.c

Titolo: Amore

Tipologia documentaria: Manoscritto

Consistenza: cc. 1-6

Autore: Palazzeschi, Aldo

Data: ante 1929 - 1957

FP36.10, 36.10.d

Titolo: Amore

Tipologia documentaria: Manoscritto

Consistenza: cc. 1-6 e c. 6 bis, rif. di c. 6 (num. mod.) + cc. 1-2 (num. mod.)

Autore: Palazzeschi, Aldo

Data: ante 1929 - 1957

FP36.10, 36.10.e

Titolo: Amore

Tipologia documentaria: Manoscritto

Consistenza: cc. 1-6

Autore: Palazzeschi, Aldo

Data: ante 1929 - 1957

FP36.10, 36.10.f

Titolo: Amore

Tipologia documentaria: Manoscritto

Consistenza: cc. 1-9

Autore: Palazzeschi, Aldo

Data: ante 1929 - 1957

\section{FP36.11}

Titolo: Bistino e il signor marchese

Consistenza: UD2

Data: ante 1934 - 1957

FP36.11, 36.11.a

Titolo: Bistino e il signor marchese

Tipologia documentaria: Manoscritto

Consistenza: cc. 1-23

Autore: Palazzeschi, Aldo

Data: ante 1934 - 1957

FP36.11, 36.11.b

Titolo: Bistino e il signor marchese

Tipologia documentaria: Manoscritto

Consistenza: cc. 1-22

Autore: Palazzeschi, Aldo

Data: ante 1934 - 1957 


\section{FP36.12}

Titolo: Lumachino

Consistenza: UD4

Data: ante 1931 - 1957

FP36.12, 36.12.a

Titolo: Lumachino

Tipologia documentaria: Manoscritto

Consistenza: cc. 1-6

Autore: Palazzeschi, Aldo

Data: ante 1931 - 1957

FP36.12, 36.12.b

Titolo: "lumachino"

Tipologia documentaria: Manoscritto

Consistenza: cc. 1-5

Autore: Palazzeschi, Aldo

Data: ante 1931 - 1957

FP36.12, 36.12.c

Titolo: Lumachino

Tipologia documentaria: Manoscritto Consistenza: cc. 1-7

Autore: Palazzeschi, Aldo

Data: ante 1931 - 1957

FP36.12, 36.12.d

Titolo: Lumachino

Tipologia documentaria: Manoscritto Stato conservazione: mutilo

Consistenza: cc. 1, 4-8 (num. mod. 1-6) Autore: Palazzeschi, Aldo

Data: ante 1931 - 1957

\section{FP36.13}

Titolo: 24 Agosto

Consistenza: UD3

Data: post 1933 - 1957

FP36.13, 36.13.a

Titolo: 24 Agosto

Tipologia documentaria: Manoscritto Consistenza: cc. 1-21

Autore: Palazzeschi, Aldo

Data: post 1933 - 1957

FP36.13, 36.13.b

Titolo: 24 Agosto

Tipologia documentaria: Manoscritto 
Consistenza: cc. 1-19

Autore: Palazzeschi, Aldo

Data: post 1933 - 1957

FP36.13, 36.13.c

Titolo: 24 Agosto

Tipologia documentaria: Manoscritto

Consistenza: cc. 1-20

Autore: Palazzeschi, Aldo

Data: post 1933 - 1957

\section{FP36.14}

Titolo: Il ricordo della moglie

Consistenza: UD3

Data: ante 1937- 1957

\section{FP36.14, 36.14.a}

Titolo: Il ricordo della moglie

Tipologia documentaria: Manoscritto

Consistenza: cc. 1-29

Autore: Palazzeschi, Aldo

Data: ante 1937 - 1957

FP36.14, 36.14.b

Titolo: Il ricordo della moglie

Tipologia documentaria: Manoscritto

Consistenza: cc. 1-24

Autore: Palazzeschi, Aldo

Data: ante 1937 - 1957

FP36.14, 36.14.c

Titolo: Il ricordo della moglie

Tipologia documentaria: Manoscritto

Consistenza: cc. 1-27

Autore: Palazzeschi, Aldo

Data: ante 1937 - 1957

\section{FP36.15}

Titolo: Lupo

Consistenza: UD4

Data: ante 1930 - 1957

FP36.15, 36.15.a

Titolo: "lupo"

Tipologia documentaria: Manoscritto

Consistenza: cc. 1-16

Autore: Palazzeschi, Aldo

Data: ante 1930 - 1957 
FP36.15, 36.15.b

Titolo: "lupo"

Tipologia documentaria: Manoscritto

Stato conservazione: interrotto

Consistenza: cc. 1-11

Autore: Palazzeschi, Aldo

Data: ante 1930 - 1957

\section{FP36.15, 36.15.c}

Titolo: «che di loro spontaneamente non avrebbe dato un"

Tipologia documentaria: Manoscritto

Stato conservazione: acefalo, anepigrafo e interrotto

Consistenza: cc. 7-13 + cc. 15-16, rif. di c. 13 (cc. 7-9 num. mod. 1-3, cc. 10-13 num. mod. 1-4 e cc. 15-16 num. mod. 1-2)

Autore: Palazzeschi, Aldo

Data: ante 1930 - 1957

\section{FP36.15, 36.15.d}

Titolo: "lupo"<(Frammento della storia di Siena) $>$

Tipologia documentaria: Manoscritto

Consistenza: cc. 1-16

Autore: Palazzeschi, Aldo

Data: ante 1930 - 1957

\section{FP36.16}

Titolo: La perfezione

Consistenza: UD4

Data: post $1927-1957$

\section{FP36.16, 36.16.a}

Titolo: <Bravo> Povero chicco!

Tipologia documentaria: Manoscritto

Consistenza: cc. 1-7

Autore: Palazzeschi, Aldo

Data: post $1927-1957$

\section{FP36.16, 36.16.b}

Titolo: La perfezione

Tipologia documentaria: Manoscritto

Consistenza: cc. 1-7

Autore: Palazzeschi, Aldo

Data: post 1927 - 1957

FP36.16, 36.16.c

Titolo: La perfezione

Tipologia documentaria: Manoscritto

Consistenza: cc. 1-7

Autore: Palazzeschi, Aldo

Data: post 1927 - 1957 
FP36.16, 36.16.d

Titolo: La perfezione

Tipologia documentaria: Manoscritto

Consistenza: cc. 1-8 (c. 8 num. mod.)

Autore: Palazzeschi, Aldo

Data: post $1927-1957$

\section{FP36.17}

Titolo: "Issimo"

Consistenza: UD5

Data: post 1926 - 1957

\section{FP36.17, 36.17.a}

Titolo: "Dacché lasciato il paese nativo era venuto"

Tipologia documentaria: Manoscritto

Stato conservazione: anepigrafo

Consistenza: cc. 1-7

Autore: Palazzeschi, Aldo

Data: post 1926 - 1957

FP36.17, 36.17.b

Titolo: «Dacché lasciato il paese nativo era venuto in» Tipologia documentaria: Manoscritto

Stato conservazione: anepigrafo

Consistenza: cc. 1-6

Autore: Palazzeschi, Aldo

Data: post 1926 - 1957

FP36.17, 36.17.c

Titolo: "Dacché lasciato il paese nativo era venuto in» Tipologia documentaria: Manoscritto

Stato conservazione: anepigrafo

Consistenza: cc. 1-6

Autore: Palazzeschi, Aldo

Data: post 1926 - 1957

FP36.17, 36.17.d

Titolo: "Issimo"

Tipologia documentaria: Manoscritto

Consistenza: cc. 1-6

Autore: Palazzeschi, Aldo

Data: post 1926 - 1957

FP36.17, 36.17.e

Titolo: "Issimo"

Tipologia documentaria: Manoscritto

Consistenza: cc. 1-6 + c. 6, rif. di c. 6 (num. mod. 7)

Autore: Palazzeschi, Aldo

Data: post 1926 - 1957 


\section{- FP37}

Titolo: Tutte le novelle

Consistenza: F27

Data: post $1929-1957$

\section{FP37.1}

Titolo: Plenilunio di primavera

Consistenza: UD4

Data: post 1940 - 1957

FP37.1, 37.1.a

Titolo: Plenilunio d'Aprile

Tipologia documentaria: Manoscritto

Stato conservazione: interrotto

Consistenza: cc. 1-14

Autore: Palazzeschi, Aldo

Data: post 1940 - 1957

\section{FP37.1, 37.1.b}

Titolo: Plenilunio d'Aprile

Tipologia documentaria: Manoscritto

Consistenza: cc. 1-18 (num. mod. di 15-17 su precedente d'autore 17-19)

Autore: Palazzeschi, Aldo

Data: post $1940-1957$

\section{FP37.1, 37.1.c}

Titolo: Plenilunio d'Aprile

Tipologia documentaria: Manoscritto

Consistenza: cc. 1-17 (num. mod. 18-21)

Autore: Palazzeschi, Aldo

Data: post $1940-1957$

FP37.1, 37.1.d

Titolo: Plenilunio d'Aprile

Tipologia documentaria: Manoscritto

Consistenza: cc. 1-20 (cc. 17-20 num. mod. 18-21) + cc. 17-20, rif. di cc. 17-20

Autore: Palazzeschi, Aldo

Data: post $1940-1957$

\section{FP37.2}

Titolo: Meriggio d'estate

Consistenza: UD8

Data: post 1938 - 1957

FP37.2, 37.2.a

Titolo: Meriggio d'estate

Tipologia documentaria: Manoscritto 
Consistenza: cc. 1-6

Autore: Palazzeschi, Aldo

Data: post 1938 - 1957

FP37.2, 37.2.b

Titolo: Meriggio d'estate

Tipologia documentaria: Manoscritto

Consistenza: cc. 1-6

Autore: Palazzeschi, Aldo

Data: post 1938 - 1957

FP37.2, 37.2.c

Titolo: Meriggio d'estate

Tipologia documentaria: Manoscritto

Consistenza: cc. 1-6

Autore: Palazzeschi, Aldo

Data: post 1938 - 1957

FP37.2, 37.2.d

Titolo: Meriggio d'estate

Tipologia documentaria: Manoscritto

Consistenza: cc. 1-8

Autore: Palazzeschi, Aldo

Data: post 1938 - 1957

FP37.2, 37.2.e

Titolo: Meriggio d'estate

Tipologia documentaria: Manoscritto Consistenza: cc. 1-9

Autore: Palazzeschi, Aldo

Data: post 1938 - 1957

FP37.2, 37.2.f

Titolo: Meriggio d'estate

Tipologia documentaria: Manoscritto Consistenza: cc. 1-8

Autore: Palazzeschi, Aldo

Data: post 1938 - 1957

FP37.2, 37.2.g

Titolo: <Arno> Meriggio d'estate

Tipologia documentaria: Manoscritto

Consistenza: cc. 1-7

Autore: Palazzeschi, Aldo

Data: post 1938 - 1957

FP37.2, 37.2.h

Titolo: Arno

Tipologia documentaria: Manoscritto

Consistenza: cc. 1-6 
Autore: Palazzeschi, Aldo

Data: ante 1938 - aprile 1938

\section{FP37.3}

Titolo: Bella difficoltà delle parole

Consistenza: UD5

Data: ante 1957 - 1957

\section{FP37.3, 37.3.a}

Titolo: «neppure con un sorriso. Date le informazioni»

Tipologia documentaria: Manoscritto

Consistenza: c. 6 (num. mod. 7)

Autore: Palazzeschi, Aldo

Data: ante 1957 - 1957

\section{FP37.3, 37.3.b}

Titolo: <Poche parole> Bella difficoltà delle parole

Tipologia documentaria: Manoscritto

Consistenza: cc. 1-5

Autore: Palazzeschi, Aldo

Data: ante $1957-1957$

\section{FP37.3, 37.3.c}

Titolo: «altro letto, il maggiore dei due apre la bocca per dire» Tipologia documentaria: Manoscritto

Consistenza: c. 4 (num. mod. 6)

Autore: Palazzeschi, Aldo

Data: ante 1957 - 1957

\section{FP37.3, 37.3.d}

Titolo: <Poche parole> Bella difficoltà delle parole

Tipologia documentaria: Manoscritto

Consistenza: cc. 1-9

Autore: Palazzeschi, Aldo

Data: ante 1957 - 1957

\section{FP37.3, 37.3.e}

Titolo: «la glicine o si arrampicano le rose ovun=que»

Tipologia documentaria: Manoscritto

Consistenza: cc. 6-10 (num. mod. 1-5)

Autore: Palazzeschi, Aldo

Data: ante 1957 - 1957

\section{FP37.4}

Titolo: Il giorno e la notte

Consistenza: UD2

Data: ante 1948 - 1957 
FP37.4, 37.4.a

Titolo: Il giorno e la notte

Tipologia documentaria: Manoscritto

Stato conservazione: mutilo

Consistenza: cc. 1-7 e c. 14 (num. mod. 8)

Autore: Palazzeschi, Aldo

Data: ante 1948 - 1957

FP37.4, 37.4.b

Titolo: Il giorno e la notte

Tipologia documentaria: Manoscritto

Consistenza: cc. 1-17

Autore: Palazzeschi, Aldo

Data: ante 1948 - 1957

\section{FP37.5}

Titolo: Kan

Consistenza: UD7

Data: ante 1938 - 1957

FP37.5, 37.5.a

Titolo: Kan

Tipologia documentaria: Manoscritto

Stato conservazione: mutilo

Consistenza: cc. $1-10+$ c. 6 , rif. di c. 6 (num. mod. 6bis)

Autore: Palazzeschi, Aldo

Data: ante 1938 - 1957

FP37.5, 37.5.b

Titolo: Kan

Tipologia documentaria: Manoscritto

Consistenza: cc. 1-11

Autore: Palazzeschi, Aldo

Data: ante 1938 - 1957

FP37.5, 37.5.c

Titolo: Kan

Tipologia documentaria: Manoscritto

Consistenza: cc. 1-11

Autore: Palazzeschi, Aldo

Data: ante 1938 - 1957

FP37.5, 37.5.d

Titolo: Kan

Tipologia documentaria: Manoscritto

Consistenza: cc. 1-13

Autore: Palazzeschi, Aldo

Data: ante 1938 - 1957 
FP37.5, 37.5.e

Titolo: Kan

Tipologia documentaria: Manoscritto

Consistenza: cc. 1-16

Autore: Palazzeschi, Aldo

Data: ante 1938 - 1957

\section{FP37.5, 37.5.f}

Titolo: "Un giorno Kan entrando, sentì nell'aria qualche cosa di in=definibile» Tipologia documentaria: Manoscritto

Stato conservazione: anepigrafo e mutilo

Consistenza: 2 cc. s. n. (num. mod. 1-2)

Autore: Palazzeschi, Aldo

Data: ante 1938 - 1957

\section{FP37.5, 37.5.g}

Titolo: "gli occhi come impaurita dal fulmine si dava a correre."

Tipologia documentaria: Manoscritto

Stato conservazione: anepigrafo e acefalo

Consistenza: cc. 8-9 (num. mod. 1-2)

Autore: Palazzeschi, Aldo

Data: ante 1938 - 1957

\section{FP37.6}

Titolo: La meccanica dell'amore

Consistenza: UD5

Data: ante 1954 - 1957

FP37.6, 37.6.a

Titolo: Amore e Scienza

Tipologia documentaria: Manoscritto

Consistenza: cc. 1-7 + c. 7, rif. del finale

Autore: Palazzeschi, Aldo

Data: ante 1954 - 1957

\section{FP37.6, 37.6.b}

Titolo: <Amore e Scienza> La meccanica dell'amore

Tipologia documentaria: Manoscritto

Consistenza: cc. 1-7 (c. 7 num. mod. 8)

Autore: Palazzeschi, Aldo

Data: ante 1954 - 1957

FP37.6, 37.6.c

Titolo: La meccanica nell'amore

Tipologia documentaria: Manoscritto

Stato conservazione: interrotto

Consistenza: cc. 1-4

Autore: Palazzeschi, Aldo

Data: ante 1954 - 1957 
FP37.6, 37.6.d

Titolo: Meccanica

Tipologia documentaria: Manoscritto

Consistenza: cc. 15-20 (num. mod. 1-6)

Autore: Palazzeschi, Aldo

Data: ante 1954 - 1957

FP37.6, 37.6.e

Titolo: Meccanica

Tipologia documentaria: Manoscritto

Consistenza: cc. 1-5

Autore: Palazzeschi, Aldo

Data: ante 1954 - 1957

\section{FP37.7}

Titolo: Quando c'è la salute

Consistenza: UD2

Data: ante 1956 - 1957

\section{FP37.7, 37.7.a}

Titolo: Quando c'è la salute

Tipologia documentaria: Manoscritto

Consistenza: cc. 1-10

Autore: Palazzeschi, Aldo

Data: ante 1956 - 1957

FP37.7, 37.7.b

Titolo: Quando c'è la salute

Tipologia documentaria: Manoscritto

Consistenza: cc. 1-11

Autore: Palazzeschi, Aldo

Data: ante 1956 - 1957

\section{FP37.8}

Titolo: Re Pomodoro

Consistenza: UD5

Data: ante 1929 - 1957

\section{FP37.8, 37.8.a}

Titolo: Re pomodoro

Tipologia documentaria: Manoscritto

Consistenza: cc. 1-21 + c. 9, rif. di c. 9 (num. mod. err. 9bis)

Autore: Palazzeschi, Aldo

Data: ante 1929 - 1957

FP37.8, 37.8.b

Titolo: Re pomodoro

Tipologia documentaria: Manoscritto 
Stato conservazione: interrotto

Consistenza: cc. 1-17

Autore: Palazzeschi, Aldo

Data: ante 1929 - 1957

\section{FP37.8, 37.8.c}

Titolo: "per lasciarla subito spaventato, quasi si fosse spinto per» Tipologia documentaria: Manoscritto

Stato conservazione: anepigrafo e acefalo

Consistenza: cc. 17-21 (num. mod. 1-5)

Autore: Palazzeschi, Aldo

Data: ante $1929-1957$

FP37.8, 37.8.d

Titolo: "[sbalor]diti di tanta fantastica bellezza, non solo, ma fu il» Tipologia documentaria: Manoscritto

Stato conservazione: anepigrafo e acefalo

Consistenza: cc. $19-20+1$ c. s. n. (num. mod. 1-3)

Autore: Palazzeschi, Aldo

Data: ante 1929 - 1957

FP37.8, 37.8.e

Titolo: Re Pomodoro

Tipologia documentaria: Manoscritto

Stato conservazione: interrotto

Consistenza: cc. 1-18

Autore: Palazzeschi, Aldo

Data: ante 1929 - 1957

\section{FP37.9}

Titolo: Giulietta e Romeo

Consistenza: UD2

Data: ante 1954 - 1957

\section{FP37.9, 37.9.a}

Titolo: Giulietta e Romeo

Tipologia documentaria: Manoscritto

Consistenza: cc. 1-7

Autore: Palazzeschi, Aldo

Data: ante 1954 - 1957

FP37.9, 37.9.b

Titolo: Giulietta e Romeo

Tipologia documentaria: Manoscritto

Consistenza: cc. 1-7

Autore: Palazzeschi, Aldo

Data: ante 1954 - 1957 
FP37.10

Titolo: La memoria di Dio

Consistenza: UD4

Data: ante 1955 - 1957

FP37.10, 37.10.a

Titolo: La memoria d'Iddio

Tipologia documentaria: Manoscritto

Stato conservazione: mutilo

Consistenza: cc. 1-3 e c. 5 (num. mod. 1-4)

Autore: Palazzeschi, Aldo

Data: ante 1955 - 1957

FP37.10, 37.10.b

Titolo: La memoria d'Iddio

Tipologia documentaria: Manoscritto

Consistenza: cc. 1-9

Autore: Palazzeschi, Aldo

Data: ante 1955 - 1957

FP37.10, 37.10.c

Titolo: La memoria d'Iddio

Tipologia documentaria: Manoscritto

Consistenza: cc. 1-8 + cc. 8-9 (num. mod. 1 e 3) e c. 8 (num. mod. 2)

Autore: Palazzeschi, Aldo

Data: ante 1955 - 1957

\section{FP37.10, 37.10.d}

Titolo: La memoria d'Iddio

Tipologia documentaria: Manoscritto

Stato conservazione: mutilo

Consistenza: cc. 1-5

Autore: Palazzeschi, Aldo

Data: ante 1955 - 1957

\section{FP37.11}

Titolo: Incontro

Consistenza: UD2

Data: ante 1954 - 1957

FP37.11, 37.11.a

Titolo: Incontro

Tipologia documentaria: Manoscritto

Consistenza: cc. 1-7

Autore: Palazzeschi, Aldo

Data: ante 1954 - 1957

FP37.11, 37.11.b

Titolo: Incontro 
Tipologia documentaria: Manoscritto

Consistenza: cc. 1-7

Autore: Palazzeschi, Aldo

Data: ante 1954 - 1957

\section{FP37.12}

Titolo: Legami ignoti

Consistenza: UD1

Data: ante 1954 - 1957

\section{FP37.12, 37.12}

Titolo: Legami ignoti

Tipologia documentaria: Manoscritto

Consistenza: cc. 1-7

Autore: Palazzeschi, Aldo

Data: ante 1954 - 1957

\section{FP37.13}

Titolo: Il Paradiso terrestre

Consistenza: UD1

Data: ante 1957 - 1957

FP37.13, 37.13

Titolo: Il Paradiso terrestre

Tipologia documentaria: Manoscritto

Stato conservazione: mutilo

Consistenza: cc.1-36 e cc. 38-44 (cc. 38-44 num. mod. 37-43)

Autore: Palazzeschi, Aldo

Data: ante 1957 - 1957

\section{FP37.14}

Titolo: Un signore solo

Consistenza: UD1

Data: ante 1949 - 1957

FP37.14, 37.14

Titolo: Un signore solo

Tipologia documentaria: Manoscritto

Consistenza: cc. 1-15

Autore: Palazzeschi, Aldo

Data: ante 1949 - 1957

\section{FP37.15}

Titolo: Madame Bouché

Consistenza: UD2

Data: ante 1957-1957 
FP37.15, 37.15.a

Titolo: Madame Bouché

Tipologia documentaria: Manoscritto

Consistenza: cc. 1-9

Autore: Palazzeschi, Aldo

Data: ante 1957 - 1957

FP37.15, 37.15.b

Titolo: Madame Bouché

Tipologia documentaria: Manoscritto

Consistenza: cc. 1-9

Autore: Palazzeschi, Aldo

Data: ante 1957 - 1957

\section{FP37.16}

Titolo: Titì... e Totò

Consistenza: UD2

Data: ante 1937 - 1957

FP37.16, 37.16.a

Titolo: Titì e Totò

Tipologia documentaria: Manoscritto

Consistenza: cc. 1-15

Autore: Palazzeschi, Aldo

Data: ante 1937-1957

FP37.16, 37.16.b

Titolo: Titì e Totò

Tipologia documentaria: Manoscritto

Consistenza: cc. 1-19

Autore: Palazzeschi, Aldo

Data: ante 1937-1957

\section{FP37.17}

Titolo: Il quarto figliolo del signor Gerolamo

Consistenza: UD3

Data: ante 1937 - 1957

FP37.17, 37.17.a

Titolo: Il quarto figliolo del signor Gerolamo

Tipologia documentaria: Manoscritto

Consistenza: cc. 1-25

Autore: Palazzeschi, Aldo

Data: ante 1937 - 1957

FP37.17, 37.17.b

Titolo: Il quarto figliolo del signor Gerolamo

Tipologia documentaria: Manoscritto 
Consistenza: cc. 1-33

Autore: Palazzeschi, Aldo

Data: ante 1937 - 1957

FP37.17, 37.17.c

Titolo: Il quarto figliolo del signor Gerolamo

Tipologia documentaria: Manoscritto

Consistenza: cc. 1-25

Autore: Palazzeschi, Aldo

Data: ante 1937- 1957

\section{FP37.18}

Titolo: Il cuore e la legge

Consistenza: UD1

Data: ante 1954 - 1957

\section{FP37.18, 37.18}

Titolo: Il cuore e la legge

Tipologia documentaria: Manoscritto

Consistenza: cc. 1-7 (c. 1 num. mod. err. 1bis) + c. 1, rif. di c. 1bis (num. mod. 1-8) Autore: Palazzeschi, Aldo

Data: ante $1954-1957$

\section{FP37.19}

Titolo: La squadraccia di Via Redentoristi

Consistenza: UD1

Data: ante 1948 - 1957

FP37.19, 37.19

Titolo: La squadraccia di Via Redentoristi

Tipologia documentaria: Manoscritto

Consistenza: cc. 1-12

Autore: Palazzeschi, Aldo

Data: ante 1948 - 1957

\section{FP37.20}

Titolo: Ricchezza

Consistenza: UD2

Data: ante 1954 - 1957

FP37.20, 37.20.a

Titolo: Ricchezza

Tipologia documentaria: Manoscritto

Consistenza: cc. 1-7

Autore: Palazzeschi, Aldo

Data: ante 1954 - 1957 
FP37.20, 37.20.b

Titolo: Ricchezza

Tipologia documentaria: Manoscritto

Consistenza: cc. 1-7

Autore: Palazzeschi, Aldo

Data: ante 1954 - 1957

\section{FP37.21}

Titolo: La porta accanto

Consistenza: UD1

Data: ante 1955 - 1957

FP37.21, 37.21

Titolo: La porta accanto

Tipologia documentaria: Manoscritto

Consistenza: cc. 1-7

Autore: Palazzeschi, Aldo

Data: ante 1955 - 1957

\section{FP37.22}

Titolo: La sora Cecchina

Consistenza: UD2

Data: ante 1956 - 1957

FP37.22, 37.22.a

Titolo: La sora Cecchina

Tipologia documentaria: Manoscritto

Stato conservazione: mutilo

Consistenza: cc. 1-7

Autore: Palazzeschi, Aldo

Data: ante 1956 - 1957

FP37.22, 37.22.b

Titolo: La sora Cecchina

Tipologia documentaria: Manoscritto

Stato conservazione: mutilo

Consistenza: cc. 1-8 (c. 8 num. mod. err. 8 bis) e c. 10 + c. 8 , rif. della c. num. mod. err. 8bis

Autore: Palazzeschi, Aldo

Data: ante 1956 - 1957

\section{FP37.23}

Titolo: Servite Domino in laetitia

Consistenza: UD2

Data: ante 1957 - 1957 
FP37.23, 37.23.a

Titolo: Servite Domino in $<L>$ laetitia Tipologia documentaria: Manoscritto Consistenza: cc. 1-10

Autore: Palazzeschi, Aldo

Data: ante 1957 - 1957

FP37.23, 37.23.b

Titolo: Servite Domino in laetitia

Tipologia documentaria: Manoscritto Consistenza: cc. 1-10

Autore: Palazzeschi, Aldo

Data: ante 1957 - 1957

\section{FP37.24}

Titolo: Silenzio

Consistenza: UD3

Data: ante 1948 - 1957

FP37.24, 37.24.a

Titolo: Silenzio

Tipologia documentaria: Manoscritto Consistenza: cc. 1-18

Autore: Palazzeschi, Aldo

Data: ante 1948 - 1957

FP37.24, 37.24.b

Titolo: Silenzio

Tipologia documentaria: Manoscritto Consistenza: cc. 1-22

Autore: Palazzeschi, Aldo

Data: ante 1948 - 1957

FP37.24, 37.24.c

Titolo: Silenzio

Tipologia documentaria: Manoscritto Consistenza: cc. 1-22

Autore: Palazzeschi, Aldo

Data: ante 1948 - 1957

\section{FP37.25}

Titolo: Fatto di cronaca

Consistenza: UD1

Data: ante 1954 - 1957

FP37.25, 37.25

Titolo: Fatto di cronaca

Tipologia documentaria: Manoscritto 
Consistenza: cc. 1-7

Autore: Palazzeschi, Aldo

Data: ante 1954 - 1957

\section{FP37.26}

Titolo: Musica proibita

Consistenza: UD3

Data: ante 1957 - 1957

\section{FP37.26, 37.26.a}

Titolo: Musica proibita

Tipologia documentaria: Manoscritto

Stato conservazione: mutilo

Consistenza: cc. 1-16 e 20-36 (cc. 20-36 num. mod. 17-34)

Autore: Palazzeschi, Aldo

Data: ante 1957 - 1957

\section{FP37.26, 37.26.b}

Titolo: Musica proibita

Tipologia documentaria: Manoscritto

Stato conservazione: mutilo

Consistenza: cc. 1-18 e 37-40 (cc. 37-40 num. mod. 19-22)

Autore: Palazzeschi, Aldo

Data: ante 1957 - 1957

FP37.26, 37.26.c

Titolo: Musica proibita

Tipologia documentaria: Manoscritto

Consistenza: cc. 1-41

Autore: Palazzeschi, Aldo

Data: ante 1957 - 1957

\section{FP37.27}

Titolo: La zia Fannì

Consistenza: UD3

Data: ante 1957 - 1957

FP37.27, 37.27

Titolo: La zia Fannì

Tipologia documentaria: Manoscritto

Consistenza: cc. 1-9 + 1 c. s. n., rif. del finale di c. 9 (num. mod. 10)

Autore: Palazzeschi, Aldo

Data: ante 1957 - 1957

\section{FP38}

Titolo: Neutrale

Consistenza: UD1 
Data: ante 1914 - $1^{\circ}$ dicembre 1914

FP38, 38

Titolo: Neutrale

Tipologia documentaria: Manoscritto

Consistenza: cc. 1-12 + 1 carta di guardia recante il titolo "Neutrale" (cassato a c. 1)

Autore: Palazzeschi, Aldo

Data: ante 1914 - $1^{\circ}$ dicembre 1914

\section{FP39}

Titolo: Don Giovanni e l'etèra

Consistenza: UD1

Data: ante 1932 - 1964

\section{FP39, 39}

Titolo: Don Giovanni e l'etèra

Tipologia documentaria: Manoscritto

Consistenza: cc. 1-7

Autore: Palazzeschi, Aldo

Data: ante $1932-1964$

\section{FP40}

Titolo: Attore mancato

Consistenza: UD4

Data: ante 1946 - 1964

FP40, 40.a

Titolo: Un attore mancato

Tipologia documentaria: Manoscritto

Stato conservazione: mutilo

Consistenza: cc. 1-16

Autore: Palazzeschi, Aldo

Data: ante 1946 - 1964

FP40, 40.b

Titolo: <Un attore mancato> «Dopo molte incertezze difronte al giudizio che si disegnava»

Tipologia documentaria: Manoscritto

Stato conservazione: anepigrafo

Consistenza: cc. 1-25

Autore: Palazzeschi, Aldo

Data: ante 1946 - 1964

FP40, 40.c

Titolo: «"Ragazzi e ragazze", diceva Luigi Rasi "Il signor»

Tipologia documentaria: Manoscritto

Stato conservazione: anepigrafo e mutilo 
Consistenza: cc. 1-11

Autore: Palazzeschi, Aldo

Data: ante 1946 - 1964

\section{FP40, 40.d}

Titolo: "a salutarla e a visitarla per nessuna ragione, soltanto"

Tipologia documentaria: Manoscritto

Stato conservazione: anepigrafo e mutilo

Consistenza: cc. 28-31 (num. mod. 26-29)

Autore: Palazzeschi, Aldo

Data: ante 1946 - 1964

\section{FP41}

Titolo: L'isola della fedeltà

Consistenza: UD2

Data: ante 1956 - 1964

\section{FP41, 41.a}

Titolo: <Fratello cane> L'isola della fedeltà

Tipologia documentaria: Manoscritto

Consistenza: cc. 1-10

Autore: Palazzeschi, Aldo

Data: ante 1956 - 1964

\section{FP41, 41.b}

Titolo: L'isola <dell'amor fedele > della fedeltà

Tipologia documentaria: Manoscritto

Consistenza: cc. 1-10 + c. 10, rif. di cc. 9-10 (num. mod. 11)

Autore: Palazzeschi, Aldo

Data: ante 1956 - 1964

\section{FP42}

Titolo: La lezione di Boldini

Consistenza: UD3

Data: ante 1964 - 1964

FP42, 42.a

Titolo: Giovanni Boldini <Sottane molto lunghe e spiriti un pochino corti> Tipologia documentaria: Manoscritto

Consistenza: cc. $1-8$

Autore: Palazzeschi, Aldo

Data: ante 1964 - 1964

FP42, 42.b

Titolo: Giovanni Boldini

Tipologia documentaria: Manoscritto

Consistenza: cc. 1-8

Autore: Palazzeschi, Aldo 
Data: ante 1964 - 1964

FP42, 42.c

Titolo: La lezione di Boldini

Tipologia documentaria: Manoscritto

Consistenza: cc. 1-8

Autore: Palazzeschi, Aldo

Data: ante 1964 - 1964

\section{FP43}

Titolo: "Sorella Morte"

Consistenza: UD5

Data: ante 1926 - 1964

FP43, 43.a

Titolo: "Sorella morte"

Tipologia documentaria: Manoscritto

Consistenza: cc. 1-6

Autore: Palazzeschi, Aldo

Data: ante 1926 - 1964

\section{FP43, 43.b}

Titolo: "Sorella morte"

Tipologia documentaria: Manoscritto

Consistenza: cc. 1-6 e 6-9 (le cc. 6-9 sono numerate a matita blu dall'autore 7-10. A c. 6 (7), il rif. di precedente c. 6 , biffato a matita blu. A seguire, fino a c. 9, la parte restante della prosa)

Autore: Palazzeschi, Aldo

Data: ante 1926 - 1964

FP43, 43.c

Titolo: "Sorella morte"

Tipologia documentaria: Manoscritto

Consistenza: cc. 1-7

Autore: Palazzeschi, Aldo

Data: ante 1926 - 1964

FP43, 43.d

Titolo: "Sorella morte"

Tipologia documentaria: Manoscritto

Consistenza: cc. 1-7

Autore: Palazzeschi, Aldo

Data: ante 1926 - 1964

FP43, 43.e

Titolo: "Sorella morte"

Tipologia documentaria: Manoscritto

Consistenza: cc. 1-7

Autore: Palazzeschi, Aldo 
Data: ante 1926 - 1964

FP44

Titolo: Il Palio

Consistenza: UD4

Data: ante 1938 - 1964

FP44, 44.a

Titolo: Il Palio

Tipologia documentaria: Manoscritto

Stato conservazione: mutilo

Consistenza: cc. 1-4 + cc. 3-5, rif. mutilo di cc. 3-4 (num. mod. 5-7)

Autore: Palazzeschi, Aldo

Data: ante 1938 - 1964

FP44, 44.b

Titolo: Il Palio

Tipologia documentaria: Manoscritto

Consistenza: cc. 1-7

Autore: Palazzeschi, Aldo

Data: ante 1938 - 1964

FP44, 44.C

Titolo: Il Palio

Tipologia documentaria: Manoscritto

Consistenza: cc. 1-7

Autore: Palazzeschi, Aldo

Data: ante 1938 - 1964

FP44, 44.d

Titolo: Il Palio

Tipologia documentaria: Manoscritto

Consistenza: cc. 1-6

Autore: Palazzeschi, Aldo

Data: ante 1938 - 1964

\section{FP45}

Titolo: La sora Rosina

Consistenza: UD1

Data: ante 1955 - 1964

FP45, 45

Titolo: La sora Rosina

Tipologia documentaria: Manoscritto

Consistenza: cc. 1-8

Autore: Palazzeschi, Aldo

Data: ante 1955 - 1964 
FP46

Titolo: L'umanità è brutta?

Consistenza: UD2

Data: ante 1955 - 1964

FP46, 46.a

Titolo: L'umanità è brutta?

Tipologia documentaria: Manoscritto

Consistenza: cc. 1-8

Autore: Palazzeschi, Aldo

Data: ante 1955 - 1964

FP46, 46.b

Titolo: L'umanità è brutta?

Tipologia documentaria: Manoscritto

Consistenza: cc. 1-8

Autore: Palazzeschi, Aldo

Data: ante 1955 - 1964

FP47

Titolo: Il paesaggio

Consistenza: UD1

Data: ante 1946 - 1947

FP47, 47

Titolo: Il paesaggio

Tipologia documentaria: Manoscritto

Consistenza: cc. 1-15

Autore: Palazzeschi, Aldo

Data: ante 1946 - 1947

FP48

Titolo: La signorina Fumaroli

Consistenza: UD2

Data: ante 1942 - 1964

FP48, 48.a

Titolo: La Signorina $<$ Mariani $>$ Fumaroli

Tipologia documentaria: Manoscritto

Consistenza: cc. 1-15 (num. mod. 37-51)

Autore: Palazzeschi, Aldo

Data: ante 1942 - 1964

FP48, 48.b

Titolo: La Signorina $<$ Mariani $><$ Bonavia $>$ Fumaroli Tipologia documentaria: Manoscritto

Consistenza: cc. 1-11

Autore: Palazzeschi, Aldo 
Data: ante 1942 - 1964

\section{FP49}

Titolo: Il 900

Consistenza: UD3

Data: ante 1955 - 1964

FP49, 49.a

Titolo: Il 900

Tipologia documentaria: Manoscritto

Stato conservazione: mutilo

Consistenza: cc. 1-7 + c. 5 , rif. di c. 5 (num. mod. 5bis)

Autore: Palazzeschi, Aldo

Data: ante 1955 - 1964

FP49, 49.b

Titolo: Il 900

Tipologia documentaria: Manoscritto

Consistenza: cc. 1-7

Autore: Palazzeschi, Aldo

Data: ante 1955 - 1964

FP49, 49.c

Titolo: Il 900

Tipologia documentaria: Manoscritto

Consistenza: cc. 1-8

Autore: Palazzeschi, Aldo

Data: ante 1955 - 1964

\section{FP50}

Titolo: Domenica parigina

Consistenza: UD1

Data: ante 1964 - 1964

\section{FP50, 50}

Titolo: "milanese"

Tipologia documentaria: Manoscritto

Consistenza: cc. 1-7

Autore: Palazzeschi, Aldo

Data: ante 1964 - 1964

\section{FP51}

Titolo: Ricordo di Vallecchi

Consistenza: UD1

Data: 18 febbraio 1946 - 1947 
FP51, 51

Titolo: Attilio Vallecchi

Tipologia documentaria: Manoscritto

Consistenza: cc. 1-6

Autore: Palazzeschi, Aldo

Data: 18 febbraio 1946 - 1947

\section{FP52}

Titolo: Carlo Marx a Firenze

Consistenza: UD1

Data: ante 1946 - 1964

\section{FP52, 52}

Titolo: Carlo Marx a Firenze

Tipologia documentaria: Manoscritto

Consistenza: cc. 1-10 (num. mod. 2-11)

Autore: Palazzeschi, Aldo

Data: ante 1946 - 1964

\section{FP53}

Titolo: "Il poema c'è"

Consistenza: UD2

Data: ante 1957 - 9 marzo 1957

FP53, 53.a

Titolo: "Il poema c'è"

Tipologia documentaria: Manoscritto

Consistenza: cc. 1-19

Autore: Palazzeschi, Aldo

Data: ante 1957 - 9 marzo 1957

\section{FP53, 53.b}

Titolo: "Il poema c'è"

Tipologia documentaria: Manoscritto

Consistenza: cc. 1-19

Autore: Palazzeschi, Aldo

Data: ante 1957 - 9 marzo 1957

\section{FP54}

Titolo: Son tifoso anch'io

Consistenza: UD1

Data: ante 1935 - $1^{\circ}$ maggio 1935

FP54, 54

Titolo: Son tifoso anch'io

Tipologia documentaria: Manoscritto

Consistenza: cc. 1-29 
Autore: Palazzeschi, Aldo

Data: ante 1935 - $1^{\circ}$ maggio 1935

\section{FP55}

Titolo: Il codice di Paul Poiret

Consistenza: UD1

Data: ante 1931 - aprile 1931

FP55, 55

Titolo: Il codice di Paul Poiret

Tipologia documentaria: Manoscritto

Consistenza: cc. 1-11

Autore: Palazzeschi, Aldo

Data: ante 1931 - aprile 1931

\section{FP56}

Titolo: Il pittore della luce elettrica

Consistenza: UD1

Data: ante 1931 - 1964

\section{FP56, 56}

Titolo: Toulouse-Lautrec. Gambe di donne e di cavalli alla luce elettrica Tipologia documentaria: Manoscritto

Consistenza: cc. 1-6

Autore: Palazzeschi, Aldo

Data: ante 1931 - 1964

\section{FP57}

Titolo: Come i fiorentini amano Firenze

Consistenza: UD1

Data: ante 1937 - 1937

\section{FP57, 57}

Titolo: Come i fiorentini amano Firenze

Tipologia documentaria: Manoscritto

Consistenza: cc. 1-6

Autore: Palazzeschi, Aldo

Data: ante 1937 - 1937

\section{FP58}

Titolo: Monsieur Debout

Consistenza: UD1

Data: ante 1945 - 1964

FP58, 58

Titolo: Monsieur Debout 
Tipologia documentaria: Manoscritto Consistenza: cc. 1-10

Autore: Palazzeschi, Aldo

Data: ante 1945 - 1964

\section{FP59}

Titolo: Le due famiglie

Consistenza: UD1

Data: ante 1921 - 1921

\section{FP59, 59}

Titolo: Le due famiglie

Tipologia documentaria: Manoscritto Stato conservazione: mutilo

Consistenza: cc. 1-10

Autore: Palazzeschi, Aldo

Data: ante 1921 - 1921

\section{FP60}

Titolo: Gaio

Consistenza: UD5

Data: ante 1951 - 1957

FP60, 60.a

Titolo: Gaio

Tipologia documentaria: Manoscritto Stato conservazione: mutilo

Consistenza: cc. 1-5

Autore: Palazzeschi, Aldo

Data: ante 1951 - 1957

FP60, 60.b

Titolo: Gaio

Tipologia documentaria: Manoscritto

Consistenza: cc. 1-9

Autore: Palazzeschi, Aldo

Data: ante 1951 - 1957

FP60, 60.c

Titolo: Gaio

Tipologia documentaria: Manoscritto

Consistenza: cc. 1-9

Autore: Palazzeschi, Aldo

Data: ante 1951 - 1957

FP60, 60.d

Titolo: Gaio

Tipologia documentaria: Manoscritto 
Consistenza: cc. 1-9

Autore: Palazzeschi, Aldo

Data: ante 1951 - 1957

FP60, 60.e

Titolo: Gaio

Tipologia documentaria: Manoscritto

Consistenza: cc. 1-9

Autore: Palazzeschi, Aldo

Data: ante 1951 - 1957

\section{FP61}

Titolo: Pompona

Consistenza: UD6

Data: ante 1938 - 1957

FP61, 61.a

Titolo: Pompona

Tipologia documentaria: Manoscritto

Consistenza: cc. 1-8

Autore: Palazzeschi, Aldo

Data: ante 1938 - 1957

FP61, 61.b

Titolo: Pompona

Tipologia documentaria: Manoscritto

Consistenza: cc. 1-11

Autore: Palazzeschi, Aldo

Data: ante 1938 - 1957

FP61, 61.c

Titolo: Pompona

Tipologia documentaria: Manoscritto

Consistenza: cc. 1-12

Autore: Palazzeschi, Aldo

Data: ante 1938 - 1957

FP61, 61.d

Titolo: Pompona

Tipologia documentaria: Manoscritto

Consistenza: cc. 1-11

Autore: Palazzeschi, Aldo

Data: ante 1938 - 1957

FP61, 61.e

Titolo: Pompona

Tipologia documentaria: Manoscritto

Consistenza: cc. 1-12

Autore: Palazzeschi, Aldo 
Data: ante 1938 - 1957

\section{FP61, 61.f}

Titolo: Pompona

Tipologia documentaria: Manoscritto

Consistenza: cc. 1-12

Autore: Palazzeschi, Aldo

Data: ante 1938-1957

\section{FP62}

Titolo: Interrogatorio della Contessa Maria

Consistenza: UD2

Data: anni Venti - anni Venti

FP62, 62.a

Titolo: Interrogatorio della Contessa Maria

Tipologia documentaria: Manoscritto

Consistenza: cc. 1-40, cc. 1-8 (num. mod. 41-48), c. 41 (num. mod. 49), 1 c. s. n.

(num. mod. 50), cc. 1-2 (num. mod. 51-52), 2 cc. s. n. (num. mod. 53-54), cc. 1-4

(num. mod. 56-59), 1 c. s. n. (num. mod. 60), cc. 41-48 (num. mod. 61-68), 3 cc. s.

n. (num. mod. 69-71), cc. 72-73 (num. mod. 72-73), 3 cc. s. n. (num. mod. 74-76),

cc. 1-11 (num. mod. 77-87), cc. 1-24 (c. 20 num. anche 17, cc. 1-24 num. mod. 88-

111, c. 93 scritta anche sul v.), 4 cc. s. n. (num. mod. 112-115), c. 24 (num. anche 5, num. mod. 116), c. 20 (num. mod. 117), c. 52a (sopra precedente 40, num. mod. 118), cc. 21-22 (num. mod. 119-120), cc. 64-65 (num. mod. 121-122) e cc. 1-127

(num. mod. 123-249)

Autore: Palazzeschi, Aldo

Data: anni Venti - anni Venti

\section{FP62, 62.b}

Titolo: Interrogatorio della Contessa Maria

Tipologia documentaria: Manoscritto

Consistenza: cc. 1-48 + 3 cc. s. n. (num. mod. 24a, 24b e 24c), cc. 1-15 (num. mod. 49-63), cc. 1-7 (num. mod. 64-70) e cc. 70-135 (num. mod. 71-136)

Autore: Palazzeschi, Aldo

Data: anni Venti - anni Venti

\section{FP63}

Titolo: Adamo ed Eva

Consistenza: UD1

Data: ante 1966 - 1966

\section{FP63, 63}

Titolo: Adamo ed Eva

Tipologia documentaria: Manoscritto

Consistenza: cc. 1-21

Autore: Palazzeschi, Aldo

Data: ante 1966 - 1966 


\section{FP64}

Titolo: Le Chiese di Roma

Consistenza: UD1

Data: 1905 - 17 agosto 1974

\section{FP64, 64}

Titolo: Le Chiese di Roma

Tipologia documentaria: Manoscritto

Consistenza: cc. 1-10

Autore: Palazzeschi, Aldo

Data: 1905 - 17 agosto 1974

\section{FP65}

Titolo: Strade fiorentine

Consistenza: UD1

Data: ante 1940 - 10 marzo 1940

\section{FP65, 65}

Titolo: Parco dei divertimenti. Strade fiorentine

Tipologia documentaria: Manoscritto

Consistenza: cc. 1-6

Autore: Palazzeschi, Aldo

Data: ante 1940 - 10 marzo 1940

\section{FP66}

Titolo: La signora dal ventaglio

Consistenza: UD2

Data: ante 1951 - 1957

\section{FP66, 66.a}

Titolo: La signora dal ventaglio

Tipologia documentaria: Manoscritto

Stato conservazione: mutilo

Consistenza: cc. 1-8 + c. 3, rif. di c. 3 (num. mod. 3bis)

Autore: Palazzeschi, Aldo

Data: ante 1951 - 1957

\section{FP66, 66.b}

Titolo: La signora dal ventaglio

Tipologia documentaria: Manoscritto

Stato conservazione: mutilo

Consistenza: cc. 1-12

Autore: Palazzeschi, Aldo

Data: ante 1951 - 1957

\section{FP67}

Titolo: «Dopo aver scritto: "mon cher Paul", Simone» 
Consistenza: UD1

Data: anni Cinquanta - anni Cinquanta

FP67, 67

Titolo: «Dopo aver scritto: "mon cher Paul", Simone»

Tipologia documentaria: Manoscritto

Stato conservazione: anepigrafo e mutilo

Consistenza: "Prologo" cc. 1-8, cc. 10-11 (c. 11 num. mod. err. 11bis) + c. 11, rif. di c. 9-10, cc. 12-18. "Michel" cc. 19-25. "André" cc. 26-29

Autore: Palazzeschi, Aldo

Data: anni Cinquanta - anni Cinquanta

\section{FP68}

Titolo: I provinciali di Parigi

Consistenza: UD2

Data: ante 1962 - 1964

FP68, 68.a

Titolo: I provinciali di Parigi

Tipologia documentaria: Manoscritto

Consistenza: cc. 1-7

Autore: Palazzeschi, Aldo

Data: ante $1962-1964$

FP68, 68.b

Titolo: «attraverso tante spine sempre rosea umanità, si»

Tipologia documentaria: Manoscritto

Stato conservazione: anepigrafo, acefalo e mutilo

Consistenza: cc. 3, 8-9

Autore: Palazzeschi, Aldo

Data: ante 1962 - 1964

\section{FP69}

Titolo: L'ottava opera di misericordia

Consistenza: UD1

Data: ante 1970 - 1970

FP69, 69

Titolo: «Dar mangiare agli affamati dar»

Tipologia documentaria: Manoscritto

Stato conservazione: anepigrafo e mutilo

Consistenza: cc. 1-3 e 5-13 (num. mod. 4-12)

Autore: Palazzeschi, Aldo

Data: ante 1970 - 1970

\section{FP70}

Titolo: Vita 
Consistenza: UD1

Data: anni Venti - anni Venti

FP70, 70

Titolo: Vita

Tipologia documentaria: Manoscritto

Consistenza: cc. 1-46

Autore: Palazzeschi, Aldo

Data: anni Venti - anni Venti

FP71

Titolo: Rosso e nero. Cronache del 1830

Consistenza: UD1

Data: marzo 1944 - maggio 1944

FP71, 71

Titolo: Rosso e nero. Cronache del 1830

Tipologia documentaria: Manoscritto

Stato conservazione: mutilo

Consistenza: C. 1 di guardia [recante il tit. «Rosso e nero | Cronache del 1830»]. Cc. 2-427 scritte su r. e v., cc. 428-429 solo su r. A c. 2v. inizia la num. sul verso, da c. [429], err. rip., a c. 860. Cc. 1-73, 75-429, [429]-430, 440 [num. err.]-533 + 533bis (ad int. di c. 533), 534 (il r. di c. 534 è bianco)-558 + 558bis (c. ripetuta), 559-808 + 808 (c. ripetuta), 560-860 e 861-898 (solo sul recto)

Autore: Palazzeschi, Aldo

Data: marzo 1944 - maggio 1944

\section{FP72}

Titolo: Il Doge

Consistenza: UD6

Data: ante 1953 - 1967

FP72, 72.a

Titolo: «Dalle prime ore della mattina nei»

Tipologia documentaria: Manoscritto

Stato conservazione: anepigrafo e mutilo

Consistenza: cc. 1-103 e 105-198 (num. mod. 104-197)

Autore: Palazzeschi, Aldo

Data: ante 1953 - 1967

FP72, 72.b

Titolo: "Dalle prime ore della mattina nei punti»

Tipologia documentaria: Manoscritto

Stato conservazione: anepigrafo

Consistenza: cc. 1-62 + 62 bis, rif. di c. 62, 63-252 + 252, rif. di c. 252 (num. mod. 252bis) e 253-254

Autore: Palazzeschi, Aldo

Data: ante 1953 - 1967 
FP72, 72.c

Titolo: «<Dalle $>$ Nelle prime ore della mattina $e<$ nei $>$ » Tipologia documentaria: Manoscritto

Stato conservazione: anepigrafo e mutilo

Consistenza: cc. 1-193, 197-[2]82 (num. mod. 194-279)

Autore: Palazzeschi, Aldo

Data: ante 1953 - 1967

FP72, 72.d

Titolo: "Nelle prime ore della mattina e dai punti»

Tipologia documentaria: Manoscritto

Stato conservazione: anepigrafo

Consistenza: cc. 1-83 + 83bis e 83tris, 84-171

Autore: Palazzeschi, Aldo

Data: ante 1953 - 1967

FP72, 72.e

Titolo: "Ma le patetiche, nostalgiche, tanto mai» Tipologia documentaria: Manoscritto

Stato conservazione: acefalo, anepigrafo e mutilo

Consistenza: cc. 156-190, 192-220 (num. mod. 1-64)

Autore: Palazzeschi, Aldo

Data: ante 1953 - 1967

\section{FP72, 72.f}

Titolo: "<fare: in nessuna epoca> una strage peg=gio" Tipologia documentaria: Manoscritto

Stato conservazione: acefalo, anepigrafo e mutilo

Consistenza: cc. 194-200 (num. mod. 1-7)

Autore: Palazzeschi, Aldo

Data: ante 1953 - 1967

\section{FP73}

Titolo: La Buona Tappa

Consistenza: UD5

Data: ante 1926 - 18 gennaio 1964

FP73, 73.a

Titolo: La $<b>$ Buona Tappa

Tipologia documentaria: Manoscritto

Consistenza: cc. 1-51

Autore: Palazzeschi, Aldo

Data: ante 1926 - 18 gennaio 1964

FP73, 73.b

Titolo: La Buona Tappa

Tipologia documentaria: Manoscritto

Stato conservazione: mutilo

Consistenza: cc. 1-39 e 41-54 (num. mod. 40-53) 
Autore: Palazzeschi, Aldo

Data: ante 1926 - 18 gennaio 1964

FP73, 73.c

Titolo: La Buona Tappa

Tipologia documentaria: Manoscritto

Consistenza: cc. 1-40 + cc. 27, 28 e 29, rif. delle cc. 27, 28, 29 (num. mod. err. 27bis, 28bis e 29bis) + c. 37bis, rif. di c. 37 (num. mod.)

Autore: Palazzeschi, Aldo

Data: ante 1926 - 18 gennaio 1964

FP73, 73.d

Titolo: La Buona Tappa

Tipologia documentaria: Fotocopia del manoscritto

Consistenza: cc. 1-44

Autore: Palazzeschi, Aldo

Data: ante 1926 - 18 gennaio 1964

FP73, 73.e

Titolo: La Buona Tappa

Tipologia documentaria: Fotocopia del dattiloscritto

Stato conservazione: mutilo

Consistenza: pp. 1-26

Autore: Palazzeschi, Aldo

Data: ante 1926 - 18 gennaio 1964

\section{FP74}

Titolo: La gondola

Consistenza: UD3

Data: ante 1956 - 1964

\section{FP74, 74.a}

Titolo: La gondola

Tipologia documentaria: Manoscritto

Consistenza: cc. 1-11 + c. 9 , rif. di c. 9 (err. num. mod. 9 bis) + c. 10, rif. di c. 10 (num. mod. 10bis)

Autore: Palazzeschi, Aldo

Data: ante 1956 - 1964

FP74, 74.b

Titolo: La gondola

Tipologia documentaria: Manoscritto

Consistenza: cc. 1-9

Autore: Palazzeschi, Aldo

Data: ante 1956 - 1964

FP74, 74.c

Titolo: La gondola

Tipologia documentaria: Manoscritto 
Consistenza: cc. 1-11

Autore: Palazzeschi, Aldo

Data: ante 1956 - 1964

\section{FP75}

Titolo: La sora Vittoria

Consistenza: UD1

Data: ante 1931 - 1964

\section{FP75, 75}

Titolo: La sora Vittoria

Tipologia documentaria: Manoscritto

Consistenza: cc. 183-228 (num. mod. 1-46)

Autore: Palazzeschi, Aldo

Data: ante 1931 - 1964

\section{FP76}

Titolo: L'amico Galletti

Consistenza: UD2

Data: ante 1960 - 1966

\section{FP76, 76.a}

Titolo: L'amico Galletti

Tipologia documentaria: Manoscritto

Stato conservazione: mutilo

Consistenza: cc. 1-19, 21-32 + c. 7, rif. di c. 7 (num. mod. 7bis)

Autore: Palazzeschi, Aldo

Data: ante 1960 - 1966

FP76, 76.b

Titolo: L'amico Galletti

Tipologia documentaria: Manoscritto

Consistenza: cc. 1-33

Autore: Palazzeschi, Aldo

Data: ante 1960 - 1966

FP77

Titolo: L'uomo del campanello

Consistenza: UD2

Data: ante 1957 - 1966

FP77, 77.a

Titolo: L'uomo del campanello

Tipologia documentaria: Manoscritto

Consistenza: cc. 1-8 + cc. 6, 7 e 8, rif. di cc. 6, 7 e 8 (num. mod. 6bis, 7bis e 8bis)

Autore: Palazzeschi, Aldo

Data: ante 1957- 1966 
FP77, 77.b

Titolo: L'uomo del campanello

Tipologia documentaria: Manoscritto

Consistenza: cc. 1-11

Autore: Palazzeschi, Aldo

Data: ante 1957 - 1966

\section{FP78}

Titolo: La donna alla finestra

Consistenza: UD1

Data: ante 1966 - 1966

\section{FP78, 78}

Titolo: La donna alla finestra

Tipologia documentaria: Manoscritto

Consistenza: cc. 1-11

Autore: Palazzeschi, Aldo

Data: ante 1966 - 1966

\section{FP79}

Titolo: I 4 cavalieri della Tazza d'oro

Consistenza: UD2

Data: ante 1958 - 1966

\section{FP79, 79.a}

Titolo: Firmino, Brunetto, Calisto e Galeazzo Tipologia documentaria: Manoscritto

Consistenza: cc. 1-8

Autore: Palazzeschi, Aldo

Data: ante 1958 - 1966

FP79, 79.b

Titolo: Firmino, Brunetto, Callisto e Galeazzo

Tipologia documentaria: Manoscritto

Consistenza: cc. 1-10

Autore: Palazzeschi, Aldo

Data: ante 1958 - 1966

\section{FP80}

Titolo: La piccola Maria

Consistenza: UD1

Data: ante 1966 - 1966

\section{FP80, 80}

Titolo: La piccola Maria

Tipologia documentaria: Manoscritto

Consistenza: cc. 1-20 
Autore: Palazzeschi, Aldo

Data: ante 1966 - 1966

\section{FP81}

Titolo: Il senso politico

Consistenza: UD4

Data: ante 1958 - 1966

\section{FP81, 81.a}

Titolo: Il gusto politico

Tipologia documentaria: Manoscritto

Consistenza: cc. 1-8

Autore: Palazzeschi, Aldo

Data: ante 1958 - 1966

\section{FP81, 81.b}

Titolo: $l l<$ gusto $>$ senso politico

Tipologia documentaria: Manoscritto

Consistenza: cc. 1-8

Autore: Palazzeschi, Aldo

Data: ante 1958 - 1966

\section{FP81, 81.c}

Titolo: «molto visibilmente un ritratto di Lauro. Qui la» Tipologia documentaria: Manoscritto

Stato conservazione: anepigrafo, acefalo e mutilo

Consistenza: cc. 6-8

Autore: Palazzeschi, Aldo

Data: ante 1958 - 1966

\section{FP81, 81.d}

Titolo: Il senso politico

Tipologia documentaria: Manoscritto

Consistenza: cc. 1-12

Autore: Palazzeschi, Aldo

Data: ante 1958 - 1966

\section{FP82}

Titolo: Una lettera d'amore

Consistenza: UD2

Data: ante 1960 - 1966

\section{FP82, 82.a}

Titolo: Una lettera d'amore

Tipologia documentaria: Manoscritto

Consistenza: cc. 1-8 + c. 5 , rif. di c. 5 (num. mod. 5bis)

Autore: Palazzeschi, Aldo

Data: ante 1960 - 1966 
FP82, 82.b

Titolo: Una lettera d'amore

Tipologia documentaria: Manoscritto

Consistenza: cc. 1-9

Autore: Palazzeschi, Aldo

Data: ante 1960 - 1966

\section{FP83}

Titolo: Non ammazzare

Consistenza: UD3

Data: ante 1962 - 1966

\section{FP83, 83.a}

Titolo: Non ammazzare

Tipologia documentaria: Manoscritto Consistenza: cc. 1-8

Autore: Palazzeschi, Aldo

Data: ante 1962 - 1966

FP83, 83.b

Titolo: Non ammazzare

Tipologia documentaria: Manoscritto Stato conservazione: mutilo

Consistenza: cc. 1-7

Autore: Palazzeschi, Aldo

Data: ante 1962 - 1966

FP83, 83.c

Titolo: Non ammazzare

Tipologia documentaria: Manoscritto

Consistenza: cc. 1-9

Autore: Palazzeschi, Aldo

Data: ante 1962 - 1966

\section{FP84}

Titolo: Il nonno

Consistenza: UD1

Data: ante 1965 - 1966

FP84, 84

Titolo: Il nonno

Tipologia documentaria: Manoscritto

Consistenza: cc. 1-5

Autore: Palazzeschi, Aldo

Data: ante 1965 - 1966 


\section{FP85}

Titolo: «No!» all'orecchio

Consistenza: UD3

Data: ante 1961 - 1966

\section{FP85, 85.a}

Titolo: «No!»<a> All'orecchio

Tipologia documentaria: Manoscritto

Consistenza: cc. 1-8 + cc. 4, 5 e 6, rif. di c. 4, 5 e 5-6 (num. mod. 4bis, 5bis, 6bis)

Autore: Palazzeschi, Aldo

Data: ante 1961 - 1966

\section{FP85, 85.b}

Titolo: «No!» all'orecchio

Tipologia documentaria: Manoscritto

Consistenza: cc. 1-8

Autore: Palazzeschi, Aldo

Data: ante 1961 - 1966

\section{FP85, 85.C}

Titolo: «No!» all'orecchio

Tipologia documentaria: Manoscritto

Consistenza: cc. 1-9

Autore: Palazzeschi, Aldo

Data: ante 1961 - 1966

\section{FP86}

Titolo: L'uomo più bello del mondo

Consistenza: UD4

Data: ante 1961-1966

FP86, 86.a

Titolo: L'uomo più bello del mondo

Tipologia documentaria: Manoscritto

Consistenza: cc. 1-7

Autore: Palazzeschi, Aldo

Data: ante 1961-1966

FP86, 86.b

Titolo: L'uomo più bello del mondo

Tipologia documentaria: Manoscritto

Consistenza: cc. 1-7

Autore: Palazzeschi, Aldo

Data: ante 1961-1966

FP86, 86.c

Titolo: L'uomo più bello del mondo Tipologia documentaria: Manoscritto Consistenza: cc. 1-7 
Autore: Palazzeschi, Aldo

Data: ante 1961-1966

FP86, 86.d

Titolo: L'uomo più bello del mondo

Tipologia documentaria: Manoscritto

Consistenza: cc. 1-11

Autore: Palazzeschi, Aldo

Data: ante 1961-1966

\section{FP87}

Titolo: «Diomio che freddo! Miodio che caldo!»

Consistenza: UD3

Data: ante 1958 - 1966

\section{FP87, 87.a}

Titolo: Che caldo!

Tipologia documentaria: Manoscritto

Consistenza: cc. 1-8 + c. 2, rif. di c. 2 (num. err. mod. 2 bis) e c. 8 , rif. di c. 8 (num. mod. 8bis)

Autore: Palazzeschi, Aldo

Data: ante 1958 - 1966

FP87, 87.b

Titolo: «mesi oscilla intorno allo zero, un po' sopra un»

Tipologia documentaria: Manoscritto

Stato conservazione: acefalo e anepigrafo

Consistenza: cc. 2-8

Autore: Palazzeschi, Aldo

Data: ante 1958 - 1966

FP87, 87.c

Titolo: <Uff! che caldo.> Mio Dio che freddo! Uff! che caldo.

Tipologia documentaria: Manoscritto

Consistenza: cc. 1-8

Autore: Palazzeschi, Aldo

Data: ante 1958 - 1966

\section{FP88}

Titolo: Il promesso sposo

Consistenza: UD3

Data: ante 1960 - 1966

\section{FP88, 88.a}

Titolo: Il promesso sposo

Tipologia documentaria: Manoscritto

Consistenza: cc. 1-8

Autore: Palazzeschi, Aldo 
Data: ante 1960 - 1966

\section{FP88, 88.b}

Titolo: Il promesso sposo

Tipologia documentaria: Manoscritto

Consistenza: cc. 1-7

Autore: Palazzeschi, Aldo

Data: ante 1960 - 1966

FP88, 88.c

Titolo: Il promesso sposo

Tipologia documentaria: Manoscritto

Consistenza: cc. 1-10

Autore: Palazzeschi, Aldo

Data: ante 1960 - 1966

\section{FP89}

Titolo: Nino e Ninetta

Consistenza: UD1

Data: ante 1966 - 1966

FP89, 89

Titolo: Nino e Ninetta

Tipologia documentaria: Manoscritto

Consistenza: cc. 1-19 + c. 1obis, rif. di c. 10

Autore: Palazzeschi, Aldo

Data: ante 1966 - 1966

\section{FP90}

Titolo: Sorelle Materassi

Consistenza: UD4

Data: 1932 - 1960

\section{FP90, 90.a}

Titolo: Sorelle Materassi

Tipologia documentaria: Manoscritto

Stato conservazione: anepigrafo e mutilo

Consistenza: Santa Maria a Coverciano, cc. 1-10 (a c. 1, di mano d'autore a mat. blu, «1 $1^{\circ}$ getto»). "Sorelle Materassi,,, cc. 1-50. Remo, cc. 1-15. Remo, cc. 1-15 (II redazione). "Palle,, cc. 1-14. < La società delle Nazioni> Teresa e Carolina stanno a vedere (tit. cassato e riscritto a mat. blu), cc. 1-11 + cc. 11bis, 11(3), 11(4) (ad int. di c. 10) e 12. Giselda canta, cc. 1-11. Teresa e Carolina stanno a vedere, Giselda canta, Niobe va a vendemmiare, cc. 1-40, 49-79. «Giselda! Niobe!», cc. 1- 26. Miss Pagan, cc. 1-40. Sepolte vive, cc. 1-16 + cc. 16 err. rip. (num. mod. 16bis) e 17-28 + cc. 22-25 (num. mod. 29-32), rif. del finale int. a c. 28.

Autore: Palazzeschi, Aldo

Data: 1932 - 1960 


\section{FP90, 90.b}

Titolo: Santa Maria a Coverciano

Tipologia documentaria: Manoscritto

Stato conservazione: anepigrafo

Consistenza: Santa Maria a Coverciano, cc. 1-11 (a c. 1, di mano d'autore, a mat. blu, «2a stesura»). "Sorelle Materassi,,, cc. 1-60. Remo, cc. 1-71. "Palle,, cc. 1-12. Teresa e Carolina stanno a vedere, Giselda canta, Niobe va a vendemmiare., cc. 1-90. «Giselda! Niobe!», cc. 1-28. «Remo non aveva mai scritto a lungo; solo aveva man=dato", cc. $1-32+$ cc. $322^{\circ}$ err. rip. (num. mod. 33) e 33-42 (num. mod. 34-43). Sepolte vive, cc. 1-36.

Autore: Palazzeschi, Aldo

Data: $1932-1960$

\section{FP90, 90.c}

Titolo: "<Per chi non lo avesse capito> Per quelli che non avessero capito dirò che» Tipologia documentaria: Manoscritto Stato conservazione: acefalo e anepigrafo

Consistenza: $«<$ Per chi non lo avesse capito $>$ Per quelli che non avessero capito dirò che», cc. 2-11. "Sorelle Materassi,,, cc. 12-63 + cc. 19-21, prec. redaz. delle cc. 19-21 [ex n. 10.a n + ex n. 10.b n + ex n.10.c n] + c. 55, prec. redaz. di c. 55 [ex n. 49.a n] + c. 58, prec. redaz. di c. 58 [ex n. 53.b n]. Remo, cc. 64-119. Palle, cc. 120128. Teresa e Carolina stanno a vedere, Giselda canta, Niobe va a vendemmiare., cc. 129-192. "Giselda! Niobe!», cc. 193-202 + cc. $2022^{\circ}$ (c. ripetuta), 203-213. «Remo non aveva mai scritto a lungo; aveva mandato", cc. 214-246. Sepolte vive, cc. 247-274.

Autore: Palazzeschi, Aldo

Data: $1932-1960$

\section{FP90, 90.d}

Titolo: «Sorelle Materassi»

Tipologia documentaria: Manoscritto

Consistenza: cc. 1-264. «Sorelle Materassi» I. Santa Maria a Coverciano, cc. 1-10. II. "Sorelle Materassi,,, cc. 11-53. «Sorelle Materassi»(1) III. Remo, cc. 54-103. <«Sorelle Materassi»(1)> IV Palle, cc. 104-111. «Sorelle Materassi»(1) V. Teresa e Carolina stanno a vedere, Giselda canta, Niobe va a vendemmiare, cc. 112-159. «Sorelle Materassi» V. Teresa e Carolina stanno a vedere, Giselda canta, Niobe va a vendemmiare, cc. 160-177. <«Sorelle Materassi»(1)> VI «Giselda! Niobe!», cc. 178-199. «Sorelle Materassi» VII. Peggy, cc. 200-237. «Sorelle Materassi»(1) VIII Sepolte vive, cc. 238-264.

Autore: Palazzeschi, Aldo

Data: $1932-1960$

FP91

Titolo: Un'avventura dei nostri giorni

Consistenza: UD2

Data: ante 1974 - 13 giugno 1974

FP91, 91.a

Titolo: «Sentiamo ripetere con insistenza» 
Tipologia documentaria: Manoscritto

Stato conservazione: anepigrafo e mutilo

Consistenza: cc. 1-2, 4 (num. mod. 3) + c. 4 , rif. di c. 4 (num. mod. 4 ), 5 cc., rif. di c. 5 (num. mod. 5-9), 3 cc., rif di c. 6 (num. mod. 10-12), 3 cc., rif. di c. 7 (num. mod. 13-15) e c. 8 (num. mod. 16)

Autore: Palazzeschi, Aldo

Data: ante 1974-13 giugno 1974

FP91, 91.b

Titolo: umana avventura

Tipologia documentaria: Manoscritto

Consistenza: cc. 1-8

Autore: Palazzeschi, Aldo

Data: ante 1974 - 13 giugno 1974

\section{FP92}

Titolo: La simpatia

Consistenza: UD2

Data: ante 1972 - 9-10 giugno 1972

FP92, 92.a

Titolo: La simpatia

Tipologia documentaria: Manoscritto

Consistenza: cc. 1-8

Autore: Palazzeschi, Aldo

Data: ante 1972 - 9-10 giugno 1972

FP92, 92.b

Titolo: La simpatia

Tipologia documentaria: Manoscritto

Stato conservazione: mutilo

Consistenza: cc. 1-4

Autore: Palazzeschi, Aldo

Data: ante 1972 - 9-10 giugno 1972

\section{FP93}

Titolo: Il Principe Bianco

Consistenza: UD1

Data: ante 1907 - 1947

FP93, 93

Titolo: Il Principe Bianco

Tipologia documentaria: Manoscritto

Consistenza: cc. 1-4

Autore: Palazzeschi, Aldo

Data: ante 1907 - 1947 


\section{FP94}

Titolo: Schizzi italofrancesi

Consistenza: UD3

Data: ante 1966 - 1966

\section{FP94, 94.a}

Titolo: Schizzi italofrancesi

Tipologia documentaria: Manoscritto

Consistenza: cc. 1-23 Un satyre au Bois, c. 1, Politesse, c. 2, Le garçon du comptoir, c. 3, Le flic, c. 4, Le clochard, cc. 5-6, La tapette, c. 7, Le cocu, c. 8, La dame au plian, cc. 9-10, La ouvreuse, c. 11, La dame du metro, cc. 12-13, Place Pigalle, cc. 14-15, La dame du Café, c. 16, Madame la consierge, cc. 17-18, Le travet à bicyclette, c. 19, Le travet à motorisé sa biciclette, c. 20, La boite à cigarettes, cc. 21-23

Autore: Palazzeschi, Aldo

Data: ante 1966 - 1966

\section{FP94, 94.b}

Titolo: Schizzi italofrancesi

Tipologia documentaria: Dattiloscritto

Consistenza: pp. 1-23 [datt.] Un satyre au Bois, p.1, Politesse, p. 2, Le garçon du comptoir, p. 3, Le flic, p. 4, Le clochard, pp. 5-6, La tapette, p. 7, Le cocu. Epitaphe, p. 8, La dame au pliant, pp. 9-10, L'ouvreuse, p. 11, La dame du métro, pp. 12-13, Place Pigalle, pp. 14-15, La dame du café, p. 16, Madame la concierge, pp. 17-18, Le travet a bicyclette, p. 19, Le travet a motorisé sa bicyclette, p. 20, La boîte a cigarettes, pp. 21-23

Autore: Palazzeschi, Aldo

Data: ante 1966 - 1966

\section{FP94, 94.C}

Titolo: Schizzi italofrancesi

Tipologia documentaria: Bozze di stampa impaginate con correzioni autografe Consistenza: cc. 7-37 (num. mod. 1-29) Un satyre au Bois, p. 7, Politesse, p. 8, Le garçon du comptoir, p. 9, Le flic, p. 10, Le clochard, pp. 11-12, La tapette, p. 13, Le cocu (epitaphe), p. 14, La dame au pliant, pp. 15-16, L'ouvreuse, p. 17, La dame du métro, pp. 18-19, Place Pigalle, pp. 20-22, La dame du Café, pp. 23-24 Madame la concierge, pp. 25-26, Le travet à bicyclette, p. 27, Le travet a motorisé sa bicyclette, p. 28, La boite à cigarettes, pp. 29-31

Autore: Palazzeschi, Aldo

Data: ante 1966 - 1966

\section{FP95}

Titolo: Cuor mio

Consistenza: UD1

Data: ante 1944 - 1968

FP95, 95

Titolo: Cuor mio

Tipologia documentaria: Manoscritto 
Consistenza: Incontro con la Musa, cc. 1-3, Gocciole, cc. 1-2, Dove sono?, cc. 1-24, Indovinello, cc. 1-4, Novembre, c. 1, Il Palatino, cc. 1-2, Notturno in Piazza San Pietro, cc. 1-3, Adamo, cc. 1-4, Ponte Garibaldi, cc. 1-4, Via Appia Antica, cc. 1-6, Santa Maria della Salute, cc. 1-2, Sacca della Misericordia, cc. 1-3, San Lazzaro degli Armeni, cc. 1-3, Calle dei Miracoli, cc. 1-7 + c. 5bis (c. ripetuta) (num. mod. 1-8)42, I marinai, cc. 1-4, Prato, cc. 1-2, Gavinana, cc. 1-2, Il grillo del Ponte Vecchio, cc. 1-5, Nella Sagrestia di San Lorenzo, cc. 1-5, Monte Ceceri, cc. 1-3, La madre, c. 1 (num. mod.), Bellagio, cc. 1-2, Rimini, cc. 1-2, Ravenna, cc. 1-2, Caprera, cc. 1-2 (num. mod), Caprera, cc. 1-2 (II redazione), Le acqueforti, cc. 1-3, Idillio campestre, cc. 1-2, Visita di Protocollo, c. 1 (num. mod.), «Essere o non essere», c. 1 (num. mod.), La ragazza di San Giovanni, c. 1 (num. mod.), Crisantemo, c. 1 (num. mod.), Compleanno, c. 1 (num. mod.), Per le vie di Calem, cc. 1-14, La mia stella tramonta, cc. 1-2, Paesaggio atomico, c. 1 (num. mod.), L'angelo ribelle, cc. 1-13, L'angelo ribelle, cc. 1-15 (II redazione), Rue de Buci, cc. 1-7, Un satyre au Bois, c. 1, Politesse, c. 1, Le garçon du comptoir, c. 1, Le flic, c. 1 (num. mod.), Le clochard, cc. 1-2, La tapette, c. 1, Le cocu (epitaphe), c. 1, Le dame au pliant, cc. 1-2, L’ouvreuse, c. 1, La dame du métro, c. 1, La dame du café, c. 1, Madame la consierge, cc. 1-2, Le travet à bicyclette, c. 1, Le travet a motorisé sa bicyclette, c. 1 (num. mod.), Place Pigalle, cc. 1-3 (c. 3, senza num. d'autore, num. mod.), La boîte à cigarettes, cc. 1-2 (vv. 1-42)

Autore: Palazzeschi, Aldo

Data: ante $1944-1968$ 


\section{- FP96}

Titolo: Nove sinfonie

Consistenza: F10

Data: 1905 - 17 agosto 1974

\section{FP96.1}

Titolo: Piazza della Libertà

Consistenza: UD6

Data: ante 1972 - 1973

FP96.1, 96.1.a

Titolo: Piazza della Libertà

Tipologia documentaria: Manoscritto

Consistenza: pp. 1-9 (num. mod.)

Autore: Palazzeschi, Aldo

Data: ante 1972 - 1973

FP96.1, 96.1.b

Titolo: Piazza della libertà

Tipologia documentaria: Manoscritto

Consistenza: cc. 1-9

Autore: Palazzeschi, Aldo

Data: ante 1972 - 1973

FP96.1, 96.1.c

Titolo: Piazza della libertà

Tipologia documentaria: Manoscritto Consistenza: cc. 1-9

Autore: Palazzeschi, Aldo

Data: ante 1972 - 1973

FP96.1, 96.1.d

Titolo: Piazza della libertà

Tipologia documentaria: Manoscritto Consistenza: cc. 1-12

Autore: Palazzeschi, Aldo

Data: ante 1972 - 1973

FP96.1, 96.1.e

Titolo: Piazza della Libertà

Tipologia documentaria: Manoscritto

Consistenza: cc. 1-11

Autore: Palazzeschi, Aldo

Data: ante $1972-1973$

FP96.1, 96.1.f

Titolo: Piazza della libertà

Tipologia documentaria: Manoscritto 
Consistenza: cc. 1-12

Autore: Palazzeschi, Aldo

Data: ante 1972 - 1973

\section{FP96.2}

Titolo: Poesia

Consistenza: UD2

Data: ante 1973 - 17 agosto 1974

FP96.2, 96.2.a

Titolo: La poesia

Tipologia documentaria: Manoscritto Consistenza: p. 10 (num. mod.)

Autore: Palazzeschi, Aldo

Data: ante 1973 - 17 agosto 1974

FP96.2, 96.2.b

Titolo: <La p > Poesia

Tipologia documentaria: Manoscritto Consistenza: p. 11 (num. mod.)

Autore: Palazzeschi, Aldo

Data: ante 1973 - 17 agosto 1974

\section{FP96.3}

Titolo: Goliardica

Consistenza: UD2

Data: ante 1973 - marzo-giugno 1974

FP96.3. 96.3.a

Titolo: Goliardica

Tipologia documentaria: Manoscritto

Consistenza: cc. 1-8

Autore: Palazzeschi, Aldo

Data: ante 1973 - marzo-giugno 1974

FP96.3, 96.3.b

Titolo: Goliardica

Tipologia documentaria: Manoscritto

Consistenza: cc. 1-6

Autore: Palazzeschi, Aldo

Data: ante 1973 - marzo-giugno 1974

\section{FP96.4}

Titolo: Gente

Consistenza: UD2

Data: ante 1973 - marzo-agosto 1974 
FP96.4, 96.4.a

Titolo: Gente

Tipologia documentaria: Manoscritto

Consistenza: cc. 1-7

Autore: Palazzeschi, Aldo

Data: ante 1973 - marzo-agosto 1974

FP96.4, 96.4.b

Titolo: Gente

Tipologia documentaria: Manoscritto

Consistenza: cc. 1-7

Autore: Palazzeschi, Aldo

Data: ante 1973 - marzo-agosto 1974

\section{FP96.5}

Titolo: I contrari

Consistenza: UD2

Data: ante 1973 - 17 agosto 1974

FP96.5, 96.5.a

Titolo: I contrari

Tipologia documentaria: Manoscritto

Consistenza: cc. 1-14

Autore: Palazzeschi, Aldo

Data: ante 1973 - 17 agosto 1974

FP96.5, 96.5.b

Titolo: I contrari

Tipologia documentaria: Manoscritto

Consistenza: cc. 1-17 (cc. 10-12 num. di mano d'autore $10 \mathrm{bis}, 102^{\circ}, 103^{\circ}$, cc. $10 \mathrm{bis}-$

17, num. mod. 11-20)

Autore: Palazzeschi, Aldo

Data: ante 1973 - 17 agosto 1974

\section{FP96.6}

Titolo: Ipocrisia

Consistenza: UD5

Data: ante 1973 - 17 agosto 1974

\section{FP96.6, 96.6.a}

Titolo: «<È virtù di tutte $>$ La virtù di tutte le virtù»

Tipologia documentaria: Manoscritto

Stato conservazione: anepigrafo

Consistenza: $1 \mathrm{c}$.

Autore: Palazzeschi, Aldo

Data: ante 1973 - 17 agosto 1974 
FP96.6, 96.6.b

Titolo: "Se tutte quelle virtù»

Tipologia documentaria: Manoscritto

Stato conservazione: anepigrafo

Consistenza: 4 cc. r. e v. s. n.

Autore: Palazzeschi, Aldo

Data: ante 1973 - 17 agosto 1974

FP96.6, 96.6.c

Titolo: Ipocrisia

Tipologia documentaria: Manoscritto

Consistenza: cc. 1-6

Autore: Palazzeschi, Aldo

Data: ante 1973 - 17 agosto 1974

FP96.6, 96.6.d

Titolo: Ipocrisia

Tipologia documentaria: Manoscritto

Consistenza: cc. 1-13

Autore: Palazzeschi, Aldo

Data: ante 1973 - 17 agosto 1974

FP96.6, 96.6.e

Titolo: Ipocrisia

Tipologia documentaria: Manoscritto Consistenza: cc. 1-15

Autore: Palazzeschi, Aldo

Data: ante 1973 - 17 agosto 1974

\section{FP96.7}

Titolo: L'Arcario

Consistenza: UD2

Data: ante 1909-1947

FP96.7, 96.7.a

Titolo: L'arcario

Tipologia documentaria: Manoscritto

Stato conservazione: mutilo

Consistenza: cc. 1-2 e 4

Autore: Palazzeschi, Aldo

Data: ante 1909 - 1947

FP96.7, 96.7.b

Titolo: $L^{\prime}<a>$ Arcario

Tipologia documentaria: Manoscritto

Consistenza: cc. 1-4

Autore: Palazzeschi, Aldo

Data: ante 1909- 1947 


\section{FP96.8}

Titolo: Lord Mailor

Consistenza: UD2

Data: ante 1909 - 1947

\section{FP96.8, 96.8.a}

Titolo: Lord Mailor

Tipologia documentaria: Manoscritto

Consistenza: cc. 1-2

Autore: Palazzeschi, Aldo

Data: ante 1909 - 1947

FP96.8, 96.8.b

Titolo: Lord Maylor

Tipologia documentaria: Manoscritto Consistenza: cc. 1-4

Autore: Palazzeschi, Aldo

Data: ante 1909 - 1947

\section{FP96.9}

Titolo: La compagnia

Consistenza: UD1

Data: 1905 - 17 agosto 1974

\section{FP96.9, 96.9}

Titolo: La compagnia

Tipologia documentaria: Manoscritto

Stato conservazione: mutilo

Consistenza: cc. 1-7

Autore: Palazzeschi, Aldo

Data: 1905 - 17 agosto 1974

\section{FP97}

Titolo: A Madre Natura

Consistenza: UD1

Data: ante 1973 - 1973

\section{FP97, 97}

Titolo: A Madre Natura

Tipologia documentaria: Manoscritto Consistenza: 1 c. s.n.

Autore: Palazzeschi, Aldo

Data: ante 1973 - 1973

\section{FP98}

Titolo: "Taccuino verde"

Consistenza: UD1 
Data: $1932-1957$

FP98, 98

Titolo: "Taccuino verde"

Tipologia documentaria: Manoscritto

Consistenza: pp. 43 (72 facciate)

Autore: Palazzeschi, Aldo

Data: $1932-1957$

\section{FP99}

Titolo: Tre diversi amici e tre liquidi diversi

Consistenza: UD1

Data: ante 1913 - 1957

\section{FP99, 99}

Titolo: Tre diversi amici e tre liquidi diversi

Tipologia documentaria: Manoscritto

Consistenza: cc. 1-4

Autore: Palazzeschi, Aldo

Data: ante 1913 - 1957

\section{FP100}

Titolo: L'angelo

Consistenza: UD1

Data: ante 1912 - 1921

FP100, 100

Titolo: L'angelo

Tipologia documentaria: Manoscritto

Consistenza: cc. 1-14

Autore: Palazzeschi, Aldo

Data: ante 1912 - 1921

\section{FP101}

Titolo: Industria

Consistenza: UD1

Data: ante 1913-1921

FP101, 101

Titolo: Industria

Tipologia documentaria: Manoscritto

Consistenza: cc. 1-18

Autore: Palazzeschi, Aldo

Data: ante 1913 - 1921 


\section{FP102}

Titolo: L'anima

Consistenza: UD1

Data: ante 1911 - 1921

FP102, 102

Titolo: L'anima

Tipologia documentaria: Manoscritto

Consistenza: cc. 1-9

Autore: Palazzeschi, Aldo

Data: ante 1911 - 1921

\section{FP103}

Titolo: Varietà

Consistenza: UD1

Data: ante 1915 - 1958

FP103, 103

Titolo: Varietà

Tipologia documentaria: Dattiloscritto

Consistenza: cc. 92-100

Autore: Palazzeschi, Aldo

Data: ante 1915 - 1958

\section{FP104}

Titolo: Equilibrio

Consistenza: UD1

Data: ante 1915 - 1958

\section{FP104, 104}

Titolo: Equilibrio

Tipologia documentaria: Dattiloscritto

Consistenza: cc. 101-114

Autore: Palazzeschi, Aldo

Data: ante 1915 - 1958

\section{FP105}

Titolo: Roma

Consistenza: UD2

Data: ante 1950 - 1960

\section{FP105, 105.a}

Titolo: Roma

Tipologia documentaria: Manoscritto

Stato conservazione: mutilo

Consistenza: Via di Monserrato, cc. 1-18. Il bambinello, cc. 19-25. Suor Giovanna Francesca, cc. 26-31. 1943, cc. 32-49. 1944, cc. 50-62. Billy-Bet, cc. 63-69 + c. 
69bis, 70-77. 1945, cc. 78-81. Checco, cc. 82-92. donna celeste, cc. 93-103. e il barbiere di Siviglia, cc. 104-107. La città del Sole, cc. 108-116. La luna, cc. 117-121. Il palazzo del numero 3, cc. 122-127. Il Duca di Rovi, cc. 128-152 + c. 152bis (num. mod.), 153-16o. Bellezza e santità, cc. 161-166. Norina, cc. 167-176 + c. 176bis, 177-196 + c. 196bis, 197-201. Si pranza alle nove: porca miseria., cc. 202-231 + c. 231bis, 232-240. L'<a>Adultera, cc. 224-249 (num. mod. 1-26). Est! Est! Est!, cc. 1-10. 1950, cc. 1-15. «Quanta luce!», cc. 16-23. <Il funerale> «Nemmeno un fiore», cc. 24-37 (cap. mutilo) + cc. 32-33, 35 e (num. mod.) 35bis (precedente stesura delle cc. 35-37). Fra Giocondo, cc. 34-57 (num. mod. 1-24)

Autore: Palazzeschi, Aldo

Data: ante 1950 - 1960

\section{FP105, 105.b}

Titolo: Roma

Tipologia documentaria: Manoscritto

Stato conservazione: interrotto

Consistenza: Roma. Via di Monserrato, cc. 1-18 (c. 4 è num. di mano d'autore «4 e 5)»). Il «<b>Bambinello», cc. 19-24. Suor Giovanna Francesca, cc. 25-30. 1943, cc. 31-46. 1944, cc. 47-56. Billy-Bet, cc. 57-69. 1945, cc. 70-72. Checco, cc. 73-81. «donna celeste», cc. 82-90. e «il barbiere di Siviglia», cc. 91-94. La città del Sole, cc. 95-102. La luna, cc. 103-107. Il palazzo del numero 3, cc. 108-113. Il Duca di Rovi, cc. 114-147. Bellezza e santità, cc. 148-152. Norina, cc. 153-184. Si pranza alle nove: porca miseria!, cc. 185-221. Adultera, cc. 222-243. Est! Est! Est!, cc. 244-253. La Duchessa di Rovi, cc. 254-255. 1950, cc. 256-270. "Quanta luce!», cc. 271-279. «Nemmeno un fiore», cc. 280-294. Fra Giocondo, cc. 295-320. Indice (num. mod. 321)

Autore: Palazzeschi, Aldo

Data: ante 1950 - 1960

\section{FP106}

Titolo: «Le memorie di Francesca Bertini»

Consistenza: UD3

Data: ante 1969 - 1969

FP106, 106.a

Titolo: «Le memorie di Francesca Bertini»

Tipologia documentaria: Manoscritto

Consistenza: cc. 1-8

Autore: Palazzeschi, Aldo

Data: ante 1969 - 1969

\section{FP106, 106.b}

Titolo: "Le memorie di Francesca Bertini»

Tipologia documentaria: Manoscritto

Consistenza: cc. 1-8

Data: ante 1969-1969

FP106, 106.c

Titolo: «Le memorie di Francesca Bertini» 
Tipologia documentaria: Manoscritto

Consistenza: cc. 1-10 (c. 10 s. n., num. mod. 10)

Autore: Palazzeschi, Aldo

Data: ante 1969 - 1969

\section{FP107}

Titolo: "Molte sono le ragioni che hanno ispirato"

Consistenza: UD1

Data: ante 1973 - 1973

\section{FP107, 107}

Titolo: «Molte sono le ragioni che hanno ispirato»

Tipologia documentaria: Manoscritto

Stato conservazione: anepigrafo

Consistenza: cc. 1-4

Autore: Palazzeschi, Aldo

Data: ante 1973 - 1973

\section{FP108}

Titolo: Alla conquista di noi stessi

Consistenza: UD1

Data: ante 1971 - 1971

\section{FP108, 108}

Titolo: «Nei primissimi giorni di Marzo del»

Tipologia documentaria: Manoscritto

Stato conservazione: anepigrafo

Consistenza: cc. 1-11

Data: ante 1971 - 1971

\section{FP109}

Titolo: Il BiBi

Consistenza: UD1

Data: ante 1960 - 1964

\section{FP109, 109}

Titolo: Il BiBi

Tipologia documentaria: Manoscritto

Consistenza: cc. 1-8 + cc. 7-8, rif. di c. 7-8 (num. mod. 7bis-8bis)

Autore: Palazzeschi, Aldo

Data: ante 1960 - 1964

\section{FP110}

Titolo: Presentazione a Il futurismo

Consistenza: UD1

Data: ante 1959 - 1959 
FP110, 110

Titolo: "Cinquant'anni costituiscono <generalmente> ordinariamente» Tipologia documentaria: Manoscritto

Stato conservazione: anepigrafo

Consistenza: cc. $1-9+$ cc. $9-10$, rif. di c. 9 (num. mod. 10-11) + 1 c. s. n., rif. di c. 10 (num. mod. 12)

Autore: Palazzeschi, Aldo

Data: ante $1959-1959$

\section{FP111}

Titolo: "Cari amici, perdonate se alle vostre domande»

Consistenza: UD2

Data: 1954 - 17 agosto 1974

FP111, 111.a

Titolo: "Cari amici, perdonate se alle vostre domande» Tipologia documentaria: Manoscritto

Stato conservazione: anepigrafo

Consistenza: cc. 1-4 (c. 4 s. n.)

Autore: Palazzeschi, Aldo

Data: 1954 - 17 agosto 1974

FP111, 111.b

Titolo: «Cari amici, perdonate se alle vostre domande» Tipologia documentaria: Manoscritto

Stato conservazione: anepigrafo

Consistenza: cc. 1-2 (num. mod.)

Autore: Palazzeschi, Aldo

Data: 1954 - 17 agosto 1974

\section{FP112}

Titolo: Alle fonti della contestazione

Consistenza: UD7

Data: ante 1969 - 1969

FP112, 112.a

Titolo: «ogni <sua> nuova audace esperienza ma»

Tipologia documentaria: Manoscritto

Stato conservazione: acefalo, anepigrafo e interrotto

Consistenza: cc. 3-9

Autore: Palazzeschi, Aldo

Data: ante 1969 - 1969

FP112, 112.b

Titolo: «Non saprei <trovare> proporre argomento più"

Tipologia documentaria: Manoscritto

Stato conservazione: anepigrafo e interrotto

Consistenza: cc. 1-10 
Autore: Palazzeschi, Aldo

Data: ante 1969 - 1969

FP112, 112.C

Titolo: "Non saprei proporre argomento più $a$ »

Tipologia documentaria: Manoscritto

Stato conservazione: anepigrafo e interrotto

Consistenza: cc. 1-6

Autore: Palazzeschi, Aldo

Data: ante 1969 - 1969

FP112, 112.d

Titolo: "Non saprei proporre un argomento più»

Tipologia documentaria: Manoscritto

Stato conservazione: anepigrafo

Consistenza: cc. 1-23

Autore: Palazzeschi, Aldo

Data: ante 1969 - 1969

FP112, 112.e

Titolo: «il cubismo una mortificazione del colore al suo apparire, mentre» Tipologia documentaria: Manoscritto

Stato conservazione: acefalo, anepigrafo e mutilo

Consistenza: cc. 13, 15-20

Autore: Palazzeschi, Aldo

Data: ante $1969-1969$

FP112, 112.f

Titolo: «Non saprei proporre un argomento più» Tipologia documentaria: Manoscritto

Stato conservazione: anepigrafo

Consistenza: cc. 1-23

Autore: Palazzeschi, Aldo

Data: ante 1969 - 1969

FP112, 112.g

Titolo: «somme di denaro, il 10 Luglio del 1913»

Tipologia documentaria: Manoscritto

Stato conservazione: acefalo, anepigrafo e mutilo

Consistenza: cc. $15-22+2$ cc. $15+$ c. 18 (precedente stesura di c. 17) + c. 21

(precedente stesura di c. 21) + 1 c. s. n. (precedente stesura di c. 22)

Autore: Palazzeschi, Aldo

Data: ante 1969 - 1969

\section{FP113}

Titolo: Storia di un'amicizia

Consistenza: UD1

Data: ante 1971 - 1971 
FP113, 113

Titolo: "La storia di Pomponio e di Cirillo"

Tipologia documentaria: Manoscritto

Stato conservazione: anepigrafo

Consistenza: cc. 2-263

Autore: Palazzeschi, Aldo

Data: ante 1971 - 1971

\section{FP114}

Titolo: Il Nuovo Istituto

Consistenza: UD1

Data: ante 1966 - 1966

FP114, 114

Titolo: «necessità, possiamo aggiungere, nel caso suo. Mentre»

Tipologia documentaria: Manoscritto

Stato conservazione: acefalo e anepigrafo

Consistenza: cc. 5-12 (num. mod. 1-8)

Autore: Palazzeschi, Aldo

Data: ante 1966 - 1966

\section{FP115}

Titolo: Premessa a Tutte le novelle

Consistenza: UD1

Data: ante 1957-1957

FP115, 115

Titolo: "La presente raccolta definitiva delle»

Tipologia documentaria: Manoscritto

Stato conservazione: anepigrafo

Consistenza: cc. 1-7

Autore: Palazzeschi, Aldo

Data: ante 1957-1957

\section{FP116}

Titolo: "1. Se vogliamo trovare un punto di appoggio"

Consistenza: UD1

Data: 1905 - 17 agosto 1974

FP116, 116

Titolo: "1. Se vogliamo trovare un punto di appoggio"

Tipologia documentaria: Manoscritto

Stato conservazione: anepigrafo

Consistenza: cc. 1-8

Autore: Palazzeschi, Aldo

Data: 1905 - 17 agosto 1974 


\section{FP117}

Titolo: Premessa alle Opere giovanili

Consistenza: UD1

Data: ante 1958 - 1958

\section{FP117, 117}

Titolo: Premessa

Tipologia documentaria: Manoscritto

Consistenza: cc. 1-6

Autore: Palazzeschi, Aldo

Data: ante 1958 - 1958

\section{FP118}

Titolo: Il processo Fenaroli come l'ha visto il grande scrittore Aldo Palazzeschi Consistenza: UD3

Data: ante 1961 - 1961

\section{FP118, 118.a}

Titolo: "sulla sua validità e sulle sue clausole»

Tipologia documentaria: Manoscritto

Stato conservazione: anepigrafo, acefalo e mutilo

Consistenza: cc. 12-17 e 19

Autore: Palazzeschi, Aldo

Data: ante 1961-1961

FP118, 118.b

Titolo: Le olimpiadi della Giustizia

Tipologia documentaria: Manoscritto

Stato conservazione: mutilo

Consistenza: cc. 1-2, 4-6 e 8-12

Autore: Palazzeschi, Aldo

Data: ante 1961- 1961

FP118, 118.c

Titolo: Le olimpiadi della Giustizia

Tipologia documentaria: Manoscritto

Consistenza: cc. 1-20

Autore: Palazzeschi, Aldo

Data: ante 1961-1961

\section{FP119}

Titolo: Un bacio puro

Consistenza: UD2

Data: ante 1957-1964

FP119, 119.a

Titolo: Un bacio puro

Tipologia documentaria: Manoscritto 
Consistenza: cc. 1-6

Autore: Palazzeschi, Aldo

Data: ante 1957-1964

\section{FP119, 119.b}

Titolo: Un bacio puro

Tipologia documentaria: Manoscritto

Consistenza: cc. 1-9 + c. 2, rif. di c. 2 (num. mod. 2bis)

Autore: Palazzeschi, Aldo

Data: ante 1957- 1964

\section{FP120}

Titolo: Alma Poesis

Consistenza: UD2

Data: ante 1961 - 1964

\section{FP120, 120.a}

Titolo: «appartamenti anche a miglior mercato e» Tipologia documentaria: Manoscritto

Stato conservazione: acefalo, anepigrafo e mutilo

Consistenza: c. 5

Data: ante 1961-1964

FP120, 120.b

Titolo: Alma Poësis

Tipologia documentaria: Manoscritto

Stato conservazione: mutilo

Consistenza: cc. 1-7

Autore: Palazzeschi, Aldo

Data: ante 1961-1964

\section{FP121}

Titolo: Il palazzo della Regina

Consistenza: UD2

Data: ante 1958 - 1964

FP121, 121.a

Titolo: Il Palazzo della Regina

Tipologia documentaria: Manoscritto

Consistenza: cc. 1-8

Autore: Palazzeschi, Aldo

Data: ante 1958 - 1964

\section{FP121, 121.b}

Titolo: Il Palazzo della Regina

Tipologia documentaria: Manoscritto

Stato conservazione: mutilo

Consistenza: cc. 1 e 5-8 + c. 1, rif. di c. 1 (num. mod. 1bis) 
Autore: Palazzeschi, Aldo

Data: ante 1958 - 1964

FP122

Titolo: I sabati da Trompeo

Consistenza: UD1

Data: ante 1958 - 1958

\section{FP122, 122}

Titolo: «Conobbi personalmente Pietro Paolo»

Tipologia documentaria: Manoscritto

Stato conservazione: anepigrafo

Consistenza: cc. 1-3

Autore: Palazzeschi, Aldo

Data: ante 1958 - 1958

\section{FP123}

Titolo: Tre italiani a Parigi

Consistenza: UD1

Data: ante 1958-1964

FP123, 123

Titolo: Tre italiani a Parigi

Tipologia documentaria: Manoscritto

Consistenza: cc. 1-8

Autore: Palazzeschi, Aldo

Data: ante 1958 - 1964

\section{FP124}

Titolo: "Quant'è bella giovinezza"

Consistenza: UD2

Data: ante 1962 - 1964

\section{FP124, 124.a}

Titolo: "Com'è bella giovinezza"

Tipologia documentaria: Manoscritto

Stato conservazione: mutilo

Consistenza: cc. 1-3 e 5-8

Autore: Palazzeschi, Aldo

Data: ante $1962-1964$

FP124, 124.b

Titolo: «serie commemorativa che gli Stati Uniti emisero» Tipologia documentaria: Manoscritto Stato conservazione: mutilo e anepigrafo Consistenza: cc. 5-8 + c. 7, rif. di c. 7 (num. mod. 7bis) Autore: Palazzeschi, Aldo 
Data: ante 1962 - 1964

\section{FP125}

Titolo: I Fiorentini e i Savoia

Consistenza: UD2

Data: ante 1960 - 1961

\section{FP125, 125.a}

Titolo: «Figli di una grande civiltà <e a quel tempo>»

Tipologia documentaria: Manoscritto

Stato conservazione: anepigrafo e acefalo

Consistenza: cc. 2-15

Autore: Palazzeschi, Aldo

Data: ante 1960 - 1961

FP125, 125.b

Titolo: I Fiorentini di cent'anni fa

Tipologia documentaria: Manoscritto

Stato conservazione: mutilo

Consistenza: cc. 1-18 + c. 1, rif. di c. 1 (num. mod. 1bis)

Autore: Palazzeschi, Aldo

Data: ante 1960 - 1961

\section{FP126}

Titolo: Ricordo di Boccioni

Consistenza: UD1

Data: ante 1957 - 1964

FP126, 126

Titolo: Ricordo di Boccioni

Tipologia documentaria: Manoscritto

Consistenza: cc. 1-8 + c. 7 (num. mod. 7bis)

Autore: Palazzeschi, Aldo

Data: ante 1957-1964

\section{FP127}

Titolo: La danza e la poesia

Consistenza: UD1

Data: 4 aprile $1961-1961$

FP127, 127

Titolo: «Debbo fare ammenda di un'imperdonabile» Tipologia documentaria: Manoscritto

Stato conservazione: anepigrafo

Consistenza: cc. $1-5+$ c. 5 , rif. di c. 5 (num. mod. 5 bis) e cc. 6-9 + c. 6, rif. di c. 6 (num. mod. 6 bis) +4 cc. 7 , rif. di c. $7+2$ cc. 8 e 1 c. s. n., rif. di c. $8+1$ c. s. n. (precedente stesura di c. 9) 
Autore: Palazzeschi, Aldo

Data: 4 aprile $1961-1961$

\section{FP128}

Titolo: Una notte a Verona

Consistenza: UD1

Data: ante 1955 - 1964

\section{FP128, 128}

Titolo: Una notte a Verona

Tipologia documentaria: Manoscritto

Consistenza: cc. 1-6 + 2 cc. 6 (num. mod. 6bis e 6tris)

Autore: Palazzeschi, Aldo

Data: ante 1955 - 1964

\section{FP129}

Titolo: Il futurismo nelle città del silenzio

Consistenza: UD2

Data: ante 1959 - 1964

\section{FP129, 129.a}

Titolo: Il futurismo nelle città del silenzio Tipologia documentaria: Manoscritto Stato conservazione: mutilo

Consistenza: cc. 1-3

Autore: Palazzeschi, Aldo

Data: ante 1959-1964

\section{FP129, 129.b}

Titolo: Il futurismo nelle città del silenzio

Tipologia documentaria: Manoscritto

Stato conservazione: mutilo

Consistenza: cc. 1-7

Autore: Palazzeschi, Aldo

Data: ante 1959 - 1964

\section{FP130}

Titolo: La camiciaia

Consistenza: UD2

Data: ante 1962 - 1964

FP130, 130.a

Titolo: La camiciaia

Tipologia documentaria: Manoscritto

Consistenza: cc. 1-6

Autore: Palazzeschi, Aldo

Data: ante 1962 - 1964 
FP130, 130.b

Titolo: La camiciaia

Tipologia documentaria: Manoscritto

Consistenza: cc. 1-7

Autore: Palazzeschi, Aldo

Data: ante 1962 - 1964

\section{FP131}

Titolo: Mio padre

Consistenza: UD1

Data: ante 1958 - 1964

\section{FP131, 131}

Titolo: Mio padre

Tipologia documentaria: Manoscritto

Consistenza: cc. 1-8

Autore: Palazzeschi, Aldo

Data: ante 1958 - 1964

\section{FP132}

Titolo: Il sor'Achille

Consistenza: UD1

Data: ante 1958 - 1964

FP132, 132

Titolo: «e una contrarietà facilmente immaginabile, riaddormenta=tosi»

Tipologia documentaria: Manoscritto

Stato conservazione: anepigrafo, acefalo e mutilo

Consistenza: cc. 2-5 e 7-8

Autore: Palazzeschi, Aldo

Data: ante 1958 - 1964

\section{FP133}

Titolo: Rosa e Azzurro

Consistenza: UD1

Data: ante 1957-1957

FP133, 133

Titolo: Rosa e Azzurro

Tipologia documentaria: Manoscritto

Consistenza: cc. $1-8+2$ cc. 8 , rif. di c. 8 (num. mod. err. 8 bis [corr. 8tris] e 8tris [corr. 8bis])

Autore: Palazzeschi, Aldo

Data: ante $1957-1957$ 


\section{FP134}

Titolo: La via gerarchica

Consistenza: UD2

Data: ante 1957-1964

\section{FP134, 134.a}

Titolo: La via gerarchica

Tipologia documentaria: Manoscritto

Stato conservazione: mutilo

Consistenza: cc. 1-7

Autore: Palazzeschi, Aldo

Data: ante 1957 - 1964

FP134, 134.b

Titolo: La via gerarchica

Tipologia documentaria: Manoscritto

Consistenza: cc. 1-8

Autore: Palazzeschi, Aldo

Data: ante 1957-1964

\section{FP135}

Titolo: I bagni di Pancaldi

Consistenza: UD1

Data: ante 1930 - 1964

\section{FP135, 135}

Titolo: Incontro col mare

Tipologia documentaria: Manoscritto

Consistenza: cc. 1-5 + c. 3 , rif. di c. 3 (num. mod. 3bis)

Autore: Palazzeschi, Aldo

Data: ante 1930 - 1964

\section{FP136}

Titolo: Poesia

Consistenza: UD1

Data: ante 1959 - 1964

FP136, 136

Titolo: Poesia

Tipologia documentaria: Manoscritto

Stato conservazione: mutilo

Consistenza: cc. 1-4 e 6-8

Autore: Palazzeschi, Aldo

Data: ante 1959 - 1964

\section{FP137}

Titolo: Il calore delle nostre tante primavere 
Consistenza: UD1

Data: ante 1968 - 1968

FP137, 137

Titolo: "Nella primavera del 1914 vi fu un'amichevole intesa»

Tipologia documentaria: Manoscritto

Stato conservazione: anepigrafo

Consistenza: cc. 1-3 + c. 3, rif. di c. 3 (num. mod. 3bis)

Autore: Palazzeschi, Aldo

Data: ante 1968 - 1968

\section{FP138}

Titolo: I provinciali a Parigi

Consistenza: UD1

Data: ante 1962 - 1964

FP138, 138

Titolo: "ventre agitato da un vento di libeccio, ebbe un attimo" Tipologia documentaria: Manoscritto

Stato conservazione: anepigrafo, acefalo e mutilo

Consistenza: cc. $4-8+$ c. 8 e 1 c. s. n., rif. di c. 8 (num. mod. 8 bis e 8 tris)

Autore: Palazzeschi, Aldo

Data: ante 1962 - 1964

\section{FP139}

Titolo: Mestre

Consistenza: UD1

Data: ante 1958 - 1958

FP139, 139

Titolo: Mestre

Tipologia documentaria: Manoscritto

Stato conservazione: mutilo

Consistenza: cc. 1-7

Autore: Palazzeschi, Aldo

Data: ante 1958 - 1958

\section{FP140}

Titolo: Lieto

Consistenza: UD2

Data: ante 1956 - 1964

FP140, 140.a

Titolo: Lieto

Tipologia documentaria: Manoscritto

Consistenza: cc. 1-9

Autore: Palazzeschi, Aldo 
Data: ante 1956 - 1964

FP140, 140.b

Titolo: Lieto

Tipologia documentaria: Manoscritto

Consistenza: cc. 1-12 + cc. 10-11, rif. di cc. 10-12 (num. mod. 1obis e 11bis)

Autore: Palazzeschi, Aldo

Data: ante 1956 - 1964

\section{FP141}

Titolo: Il naso

Consistenza: UD1

Data: ante 1960 - 1964

\section{FP141, 141}

Titolo: Il naso

Tipologia documentaria: Manoscritto

Consistenza: cc. 1-8 + cc. 7-8, rif. di cc. 7-8

Autore: Palazzeschi, Aldo

Data: ante 1960 - 1964

\section{FP142}

Titolo: "Non so se gli italiani ricordino»

Consistenza: UD2

Data: ante 1967 - 1967

FP142, 142.a

Titolo: Non so se gli italiani ricordino Tipologia documentaria: Manoscritto Consistenza: 1 c. s. n. (num. mod. 1)

Autore: Palazzeschi, Aldo

Data: ante 1967 - 1967

FP142, 142.b

Titolo: Non so se gli italiani ricordino Tipologia documentaria: Manoscritto Consistenza: 1 c. s. n. (num. mod. 2)

Autore: Palazzeschi, Aldo

Data: ante 1967 - 1967

\section{FP143}

Titolo: La tromba

Consistenza: UD1

Data: 1905 - 17 agosto 1974

FP143, 143

Titolo: La tromba 
Tipologia documentaria: Manoscritto

Consistenza: c. 1, con annotazione dell'autore che la numera come 67-68

Autore: Palazzeschi, Aldo

Data: 1905 - 17 agosto 1974

\section{FP144}

Titolo: «Rachilde, p. 141»

Consistenza: UD1

Data: post 1912 - 17 agosto 1974

\section{FP144, 144}

Titolo: «Rachilde, p. 141»

Tipologia documentaria: Manoscritto

Consistenza: $1 \mathrm{c}$.

Autore: Palazzeschi, Aldo

Data: post 1912 - 17 agosto 1974

\section{FP145}

Titolo: "Taccuino rosso"

Consistenza: UD1

Data: 1960 - 1968

\section{FP145, 145}

Titolo: "Taccuino rosso"

Tipologia documentaria: Manoscritto

Consistenza: pp. 9 (16 facciate)

Autore: Palazzeschi, Aldo

Data: $1960-1968$

\section{FP146}

Titolo: Il ritratto della regina

Consistenza: UD1

Data: ante 1948 - 1957

\section{FP146, 146}

Titolo: Il ritratto della regina

Tipologia documentaria: Manoscritto

Stato conservazione: mutilo

Consistenza: cc. 1-7

Autore: Palazzeschi, Aldo

Data: ante 1948 - 1957

\section{FP147}

Titolo: "Un giorno lo si vide equipaggiato di tutto"

Consistenza: UD1

Data: ante 1964 - 1964 
FP147, 147

Titolo: "Un giorno lo si vide equipaggiato di tutto»

Tipologia documentaria: Manoscritto

Stato conservazione: anepigrafo, acefalo e mutilo

Consistenza: c. 7

Autore: Palazzeschi, Aldo

Data: ante 1964 - 1964

\section{FP148}

Titolo: "[per] primo e sentii gridare dal fondo, lascia, non vedi che» Consistenza: UD1

Data: 1905 - 17 agosto 1974

\section{FP148, 148}

Titolo: «[per] primo e sentii gridare dal fondo, lascia, non vedi che» Tipologia documentaria: Manoscritto

Stato conservazione: anepigrafo, acefalo e mutilo

Consistenza: 1 c. s. n.

Autore: Palazzeschi, Aldo

Data: 1905 - 17 agosto 1974 


\section{Fondo Acquisizioni Centro di Studi Aldo Palazzeschi}

Serie AP 6.4

Titolo: Carte d'autore

Consistenza: S1

Data: 1928 - 1972

Contiene i manoscritti e i dattiloscritti di Aldo Palazzeschi che, staccatisi in periodi diversi dal corpus delle carte palazzeschiane, sono stati successivamente acquisiti dal Centro di Studi Aldo Palazzeschi.

\section{FPna1}

Titolo: La signorina Mariani

Consistenza: UD2

Data: 23 luglio 1942 - 1942

FPna1, 1.a

Titolo: La signorina Mariani

Tipologia documentaria: Manoscritto

Consistenza: cc. 1-12

Autore: Palazzeschi, Aldo

Data: 23 luglio 1942 - 1942

FPna1, 1.b

Titolo: La signorina Mariani

Tipologia documentaria: Dattiloscritto

Consistenza: pp. 1-5

Autore: Palazzeschi, Aldo

Data: 23 luglio $1942-1942$

\section{FPna2}

Titolo: Via delle cento stelle

Consistenza: UD1

Data: ante 1968 - 1972

FPna2, 2

Titolo: Cacherelli 100

Tipologia documentaria: Manoscritto

Consistenza: cc. 1-10o + 1 c. di frontespizio s. n.

Autore: Palazzeschi, Aldo

Data: ante 1968 - 1972 


\section{FPna3}

Titolo: Via Appia Antica

Consistenza: UD1

Data: ante 1959 - 1968

\section{FPna3, 3}

Titolo: Via Appia Antica

Tipologia documentaria: Manoscritto

Consistenza: cc. 1-5

Autore: Palazzeschi, Aldo

Data: ante 1959 - 1968

\section{FPna4}

Titolo: Stampe dell'80o

Consistenza: UD1

Data: ante 1932 - febbraio 1932

\section{FPna4, 4}

Titolo: Stampe dell'80o

Tipologia documentaria: Manoscritto

Consistenza: cc. 1-221, Dedica, c. 1, I. Una casa per me, cc. 1-8, II. Le nonne, cc.

9-15, III. La sora Parisina, cc. 16-23, IV. Che cosa era entrato in casa?, cc. 24-32,

V. Vecchie inglesi, cc. 33-56, VI. La sor' Isabella, cc. 57-69, VII. La sora Sofia, cc.

70-80, VIII. L’incendiario, cc. 81-88, IX. I fiori della libertà, cc. 89-110, X. Il teatro

Pagliano, cc. 111-124, XI. "Mi darete di più", cc. 125-146, XII. Don Giovanni e

l'etèra, cc. 147-152, XIII. La sora Vittoria, cc. 153-187, XIV. I bagni di Pancaldi, cc.

188-216, XV. Inverno, cc. 217-221

Autore: Palazzeschi, Aldo

Data: ante 1932 - febbraio 1932

\section{FPna5}

Titolo: Paesaggio

Consistenza: UD1

Data: ante 1928 - novembre 1928

\section{FPna5, 5}

Titolo: Paesaggio

Tipologia documentaria: Manoscritto

Consistenza: c. 1

Autore: Palazzeschi, Aldo

Data: ante 1928 - novembre 1928

\section{FPna6}

Titolo: Son tifoso anch'io

Consistenza: UD1

Data: ante 1934 - 1934 
FPna6, 6

Titolo: Son tifoso anch'io

Tipologia documentaria: Manoscritto

Consistenza: cc. 1-34

Autore: Palazzeschi, Aldo

Data: ante 1934 - 1934 



\section{Fondo "Carte del Signorino"}

\section{Serie AP 2.3}

Titolo: Carte d'autore

Consistenza: S1

Data: 1915 - 17 agosto 1974

Contiene i manoscritti (“Carte del Signorino") conservati dalla fedele domestica Plebe (Margherita) Bellocchio negli anni romani di Aldo

Palazzeschi

\section{FBE1}

Titolo: $\mathrm{Su}$

Consistenza: UD1

Data: ante 1915 - 15 febbraio 1915

FBE1, 1

Titolo: $\mathrm{Su}$

Tipologia documentaria: Manoscritto

Consistenza: c. 1

Autore: Palazzeschi, Aldo

Data: ante 1915 - 15 febbraio 1915

\section{FBE2}

Titolo: L'Arcario

Consistenza: UD1

Data: post 1909 - ante 1947

FBE2, 2

Titolo: L'Arcario

Tipologia documentaria: Manoscritto

Stato conservazione: mutilo

Consistenza: cc. 1-3

Autore: Palazzeschi, Aldo

Data: post 1909 - ante 1947

\section{FBE3}

Titolo: Torre Burla

Consistenza: UD1

Data: post 1907 - ante 1947

FBE3, 3

Titolo: Torre Burla 
Tipologia documentaria: Manoscritto

Consistenza: cc. 1-3

Autore: Palazzeschi, Aldo

Data: post 1907 - ante 1947

\section{FBE4}

Titolo: «1. Un curioso scrittore»

Consistenza: UD1

Data: anni Settanta - 17 agosto 1974

\section{FBE4, 4.a}

Titolo: "1. Un curioso scrittore»

Tipologia documentaria: Manoscritto

Stato conservazione: anepigrafo e mutilo

Consistenza: c. 1

Autore: Palazzeschi, Aldo

Data: anni Settanta - 17 agosto 1974

\section{FBE4, 4.b}

Titolo: «1. Un curioso scrittore»

Tipologia documentaria: Manoscritto

Stato conservazione: anepigrafo

Consistenza: cc. 1-4

Autore: Palazzeschi, Aldo

Data: anni Settanta - 17 agosto 1974

\section{FBE5}

Titolo: Correzioni a "Via delle Cento Stelle"

Consistenza: UD1

Data: ante 1972 - 1972

\section{FBE5, 5}

Titolo: Correzioni a "Via delle Cento Stelle"

Tipologia documentaria: Manoscritto

Consistenza: c. 1

Autore: Palazzeschi, Aldo

Data: ante 1972 - 1972

\section{FBE6}

Titolo: Filo diretto Palazzeschi-Sanguineti. Sull'avanguardia

Consistenza: UD1

Data: gennaio 1967 - ottobre 1967

FBE6, 6

Titolo: "Caro Sanguineti, anzitutto mi voglia»

Tipologia documentaria: Manoscritto

Stato conservazione: anepigrafo 
Consistenza: cc. 1-3

Autore: Palazzeschi, Aldo

Data: gennaio 1967 - ottobre 1967

\section{FBE7}

Titolo: «Aldo Palazzeschi: nato a Firenze il 2 Febbraio 1885"

Consistenza: UD1

Data: 1958

\section{FBE7, 7}

Titolo: "Aldo Palazzeschi: nato a Firenze il 2 Febbraio 1885"

Tipologia documentaria: Manoscritto

Stato conservazione: anepigrafo

Consistenza: 1 c. (r. e v.)

Autore: Palazzeschi, Aldo

Data: 1958

\section{FBE8}

Titolo: "Molte sono le ragioni che hanno ispirato"

Consistenza: UD1

Data: ante 1973 - 1973

\section{FBE8, 8}

Titolo: «Molte sono le ragioni che hanno ispirato»

Tipologia documentaria: Manoscritto

Stato conservazione: anepigrafo

Consistenza: cc. 1-3

Autore: Palazzeschi, Aldo

Data: ante 1973 - 1973

\section{FBE9}

Titolo: «per sua irremovibile consuetudine»

Consistenza: UD1

Data: anni Settanta - 17 agosto 1974

FBE9, 9

Titolo: «per sua irremovibile consuetudine»

Tipologia documentaria: Manoscritto

Stato conservazione: acefalo, anepigrafo e mutilo

Consistenza: c. 1

Autore: Palazzeschi, Aldo

Data: anni Settanta - 17 agosto 1974 



\section{Fondo Valentino Brosio}

\section{Serie AP 3.3}

Titolo: Carte d'autore

Consistenza: S1

Data: 1949 - 1960

Contiene il manoscritto dell'Introduzione di Aldo Palazzeschi all'opera di Valentino Brosio "Porcellane e maioliche italiane dell'Ottocento" (Milano, Vallardi, 1960, pp. 5-8), due dattiloscritti per la sceneggiatura del film "Ti ritroverò" (1949) e un appunto vergato a mano.

\section{FBR1}

Titolo: «Nell'anno di grazia 1756 la Marchesa di» Consistenza: UD1

Data: ante 1960 - 1960

FBR1, 1

Titolo: «Nell'anno di grazia 1756 la Marchesa di» Tipologia documentaria: Manoscritto Consistenza: cc. 1-9.

Autore: Palazzeschi, Aldo

Data: ante 1960 - 1960

\section{FBR2}

Titolo: Il marito scomparso

Consistenza: UD2

Data: ante 1949 - 1949

FBR2, 2.a

Titolo: Il marito scomparso

Tipologia documentaria: Dattiloscritto

Consistenza: pp. 1-2, 4-8

Autore: Brosio, Valentino; Palazzeschi, Aldo

Data: ante 1949 - 1949

FBR2, 2.b

Titolo: Il marito scomparso

Tipologia documentaria: Dattiloscritto

Stato conservazione: mutilo con correzioni a penna

Consistenza: pp. 1-16, 19-36

Autore: Brosio, Valentino; Palazzeschi, Aldo

Data: ante 1949 - 1949 
104 Fondo Valentino Brosio

FBR2, 2.c

Titolo: «Voglio mio marito»

Tipologia documentaria: Manoscritto

Consistenza: c. 1

Autore: Palazzeschi, Aldo

Data: ante 1949 - 1949 


\section{Fondo Alberto Perrini}

\section{Serie AP 4.3}

Titolo: Carte d'autore

Consistenza: S1

Data: 1954 - 6 giugno 1973

Contiene materiali relativi alla riduzione teatrale del romanzo "Roma" di Aldo Palazzeschi e Alberto Perrini e l'adattamento radiofonico curato da Antoinette Riva.

\section{FPE1}

Titolo: Roma

Consistenza: UD1

Data: ante 1954 - aprile 1955

FPE1, 1

Titolo: "Roma"

Tipologia documentaria: Dattiloscritto

Consistenza: pp. 1-79

Autore: Palazzeschi, Aldo; Perrini, Alberto

Data: ante 1954 - aprile 1955

\section{FPE2}

Titolo: Scaletta per l'adattamento e la riduzione teatrale del romanzo "Roma" di Palazzeschi

Consistenza: UD1

Data: ante 1954 - 22 agosto 1954

FPE2, 2 (ex n. 24)

Titolo: Scaletta per l'adattamento e la riduzione teatrale del romanzo "Roma" di Palazzeschi

Tipologia documentaria: Dattiloscritto

Consistenza: cc. 1-4

Autore: Perrini, Alberto

Data: ante 1954 - 22 agosto 1954

\section{FPE3}

Titolo: La riduzione teatrale di "Roma" (per il programma di sala)

Consistenza: UD1

Data: ante 1955 - 5 gennaio 1955 
FPE3, 3 (ex n. 5o)

Titolo: La riduzione teatrale di "Roma" (per il programma di sala) Tipologia documentaria: Dattiloscritto

Consistenza: c. 1

Autore: Perrini, Alberto

Data: ante 1955 - 5 gennaio 1955

\section{FPE4}

Titolo: Testo della trasmissione radiofonica (RAI-Roma)

Consistenza: UD1

Data: ante 1954 - 1954

\section{FPE4, 4 (ex n. 65)}

Titolo: Testo della trasmissione radiofonica (RAI-Roma)

Tipologia documentaria: Dattiloscritto

Consistenza: cc. 1-6

Autore: Perrini, Alberto

Data: ante 1954 - 1954

\section{FPE5}

Titolo: Palazzeschi - Roma 1950 (adattamento radiofonico francese) Consistenza: UD1

Data: 12 ottobre 1972 - 6 giugno 1973

FPE5, 5 (ex n. 71)

Titolo: Palazzeschi - Roma 1950 (adattamento radiofonico francese) Tipologia documentaria: Dattiloscritto

Consistenza: pp. 1-63

Autore: Riva, Antoinette

Data: 12 ottobre 1972 - 6 giugno 1973 



\section{Carte d'autore}

La collana intende valorizzare e diffondere, in campo nazionale e internazionale, la pubblicazione in formato digitale (Open Access) di inventari, cataloghi, mostre, studi e ricerche promosse dal Centro di Studi "Aldo Palazzeschi" nell'ambito del progetto Carte d'autore online.

1 presente lavoro intende fornire l'inventario dei manoscritti (serie Carte d'autore) conservati in cinque fondi dell'archivio del Centro di Studi «Aldo Palazzeschi» dell'Università di Firenze: Fondo «Aldo Palazzeschi»; Fondo «Acquisizioni Centro di Studi Aldo Palazzeschi»; Fondo "Carte del Signorino"; Fondo «Valentino Brosio»; Fondo «Alberto Perrini».

La parte più cospicua della documentazione è costituita dagli autografi palazzeschiani, a cui si aggiungono, nei fondi «Valentino Brosio»e «Alberto Perrini», alcuni scritti di Brosio, Perrini e Antoinette Riva (rielaborazioni di opere di Palazzeschi, autorizzate dallo scrittore o realizzate con la sua collaborazione).

La documentazione copre un arco cronologico che va dall'esordio poetico di Palazzeschi, nel 1905, anno di pubblicazione della raccolta I cavalli bianchi, al 1974, anno della morte.

SIMONE MAGHERINI è docente di Letteratura italiana presso l'Università di Firenze. Si è interessato in particolare alla cultura letteraria otto-novecentesca e allo studio dei protagonisti dell'avanguardia storica, con ricerche d'archivio, edizioni di carteggi, indagini sulle fonti, interventi sul significato della memoria dantesca e leopardiana nella poesia del Novecento. Attualmente si dedica allo studio delle molteplici forme della comunicazione letteraria sei-settecentesca e ottocentesca, tra la scuola galileiana e la stagione risorgimentale. Sul versante delle applicazioni informatiche alle discipline umanistiche ha progettato e realizzato Carte d'autore online. 